\title{
THE COS/UVES ABSORPTION SURVEY OF THE MAGELLANIC STREAM. I. ONE-TENTH SOLAR ABUNDANCES ALONG THE BODY OF THE STREAM*
}

\author{
Andrew J. Fox ${ }^{1}$, Philipp Richter ${ }^{2,3}$, Bart P. WAKkeR ${ }^{4}$, Nicolas Lehner ${ }^{5}$, J. Christopher HowK ${ }^{5}$, \\ Nadya Ben BeKhti ${ }^{6}$, Joss Bland-Hawthorn ${ }^{7}$, and StePhen LuCas ${ }^{8}$ \\ ${ }^{1}$ Space Telescope Science Institute, 3700 San Martin Drive, Baltimore, MD 21218, USA; afox@ stsci.edu \\ 2 Institut für Physik und Astronomie, Universität Potsdam, Haus 28, Karl-Liebknecht-Str. 24/25, D-14476 Potsdam, Germany \\ ${ }^{3}$ Leibniz-Institut für Astrophysik Potsdam (AIP), An der Sternwarte 16, D-14482 Potsdam, Germany \\ ${ }^{4}$ Department of Astronomy, University of Wisconsin-Madison, 475 North Charter St., Madison, WI 53706, USA \\ ${ }^{5}$ Department of Physics, University of Notre Dame, 225 Nieuwland Science Hall, Notre Dame, IN 46556, USA \\ ${ }^{6}$ Argelander-Institut für Astronomie, Universität Bonn, Auf dem Hügel 71, D-53121 Bonn, Germany \\ ${ }^{7}$ Institute of Astronomy, School of Physics, University of Sydney, NSW 2006, Australia \\ ${ }^{8}$ Department of Physics and Astronomy, University College London, Gower Street, London WC1E 6BT, UK \\ Received 2012 December 21; accepted 2013 April 11; published 2013 July 12
}

\begin{abstract}
The Magellanic Stream (MS) is a massive and extended tail of multi-phase gas stripped out of the Magellanic Clouds and interacting with the Galactic halo. In this first paper of an ongoing program to study the Stream in absorption, we present a chemical abundance analysis based on HST/COS and VLT/UVES spectra of four active galactic nuclei (RBS 144, NGC 7714, PHL 2525, and HE 0056-3622) lying behind the MS. Two of these sightlines yield good MS metallicity measurements: toward RBS 144 we measure a low MS metallicity of $[\mathrm{S} / \mathrm{H}]=[\mathrm{S} \mathrm{II} / \mathrm{HI}]=$ $-1.13 \pm 0.16$ while toward NGC 7714 we measure $[\mathrm{O} / \mathrm{H}]=[\mathrm{O} \mathrm{I} / \mathrm{HI}]=-1.24 \pm 0.20$. Taken together with the published MS metallicity toward NGC 7469, these measurements indicate a uniform abundance of $\approx 0.1$ solar along the main body of the Stream. This provides strong support to a scenario in which most of the Stream was tidally stripped from the SMC $\approx 1.5-2.5 \mathrm{Gyr}$ ago (a time at which the SMC had a metallicity of $\approx 0.1$ solar), as predicted by several $N$-body simulations. However, in Paper II of this series, we report a much higher metallicity $(\mathrm{S} / \mathrm{H}=$ 0.5 solar) in the inner Stream toward Fairall 9, a direction sampling a filament of the MS that Nidever et al. claim can be traced kinematically to the Large Magellanic Cloud, not the Small Magellanic Cloud. This shows that the bifurcation of the Stream is evident in its metal enrichment, as well as its spatial extent and kinematics. Finally we measure a similar low metallicity $[\mathrm{O} / \mathrm{H}]=[\mathrm{O} \mathrm{I} / \mathrm{HI}]=-1.03 \pm 0.18$ in the $v_{\mathrm{LSR}}=150 \mathrm{~km} \mathrm{~s}^{-1}$ cloud toward HE 0056-3622, which belongs to a population of anomalous velocity clouds near the south Galactic pole. This suggests these clouds are associated with the Stream or more distant structures (possibly the Sculptor Group, which lies in this direction at the same velocity), rather than tracing foreground Galactic material.
\end{abstract}

Key words: Galaxy: evolution - Galaxy: halo - ISM: abundances - Magellanic Clouds - ultraviolet: ISM

Online-only material: color figures

\section{INTRODUCTION}

Satellite interactions are one of the important mechanisms by which spiral galaxies acquire gas and sustain their ongoing star formation. These interactions generate extended tidal gas streams, which must pass through the galaxies' multi-phase halos in order to reach their disks. The gas brought in by tidal streams, and other processes including cold and hot accretion, clearly plays an important role in galaxy evolution (see Putman et al. 2012), but the relative importance of these processes, and the detailed manner in which gas enters galaxies, is poorly known. The extended halo of the Milky Way (MW), which contains a well-mapped population of high-velocity clouds (HVCs) and is pierced by hundreds of quasar sightlines, represents an ideal locale to investigate these galaxy feeding processes.

The Magellanic Stream (MS) stands as the most striking example of a satellite interaction in the Galactic neighborhood.

\footnotetext{
* Based on observations taken under program 12604 of the NASA/ESA Hubble Space Telescope, obtained at the Space Telescope Science Institute, which is operated by the Association of Universities for Research in Astronomy, Inc., under NASA contract NAS 5-26555, and under proposal ID 085.C-0172(A) with the Ultraviolet and Visual Echelle Spectrograph (UVES) on the Very Large Telescope (VLT) Unit 2 (Kueyen) operated by the European Southern Observatory (ESO) at Paranal, Chile.
}

Massive $\left(1.5-5 \times 10^{8} M_{\odot}\right.$ in $\mathrm{HI}$, depending on its distance; Mathewson et al. 1974; Brüns et al. 2005; Nidever et al. 2010; Besla et al. 2012), extended $\left(\approx 140^{\circ}\right.$ long, or $\approx 200^{\circ}$ when including the Leading Arm; Hulsbosch \& Wakker 1988; Brüns et al. 2005; Nidever et al. 2010), and of low-metallicity $(\approx 0.1-0.5$ solar; Lu et al. 1994; Gibson et al. 2000; Fox et al. 2010; this paper), the MS represents a prime opportunity to study the properties, origin, and fate of a nearby gaseous stream. Discovered in $21 \mathrm{~cm}$ emission (Dieter 1971; Wannier \& Wrixon 1972), the Stream exhibits a multi-phase structure including cool molecular cores (Richter et al. 2001), cold neutral (Matthews et al. 2009) and warm neutral (Brüns et al. 2005) gas, and regions of warm-ionized (Lu et al. 1994, 1998; Weiner \& Williams 1996; Putman et al. 2003a) and highly-ionized (Sembach et al. 2003; Fox et al. 2005b, 2010) plasma.

Substantial theoretical and simulational work has gone into understanding the Stream's origin and fate. The favored origin mechanisms are tidal stripping (Fujimoto \& Sofue 1976; Murai \& Fujimoto 1980; Lin \& Lynden-Bell 1982; Lin et al. 1995; Gardiner et al. 1994; Gardiner \& Noguchi 1996; Connors et al. 2006; Besla et al. 2010, 2012; Diaz \& Bekki 2011a, 2012) and ram-pressure stripping (Meurer et al. 1985; Moore \& Davis 1994; Mastropietro et al. 2005; Diaz \& Bekki 2011b), with perhaps some contribution from feedback from Large Magellanic 
Table 1

Sightline Properties and Details of Observations

\begin{tabular}{|c|c|c|c|c|c|c|c|c|c|c|c|c|}
\hline \multirow[t]{2}{*}{ Target } & \multirow[t]{2}{*}{ Type } & \multirow{2}{*}{$\begin{array}{c}l \\
\left({ }^{\circ}\right)\end{array}$} & \multirow{2}{*}{$\begin{array}{c}b \\
\left({ }^{\circ}\right)\end{array}$} & \multirow{2}{*}{$\begin{array}{c}\alpha_{\mathrm{SMC}}{ }^{\mathrm{a}} \\
\left(^{\circ}\right)\end{array}$} & \multirow{2}{*}{$\begin{array}{c}v_{0}(\mathrm{HI})_{\mathrm{MS}}{ }^{\mathrm{b}} \\
\left(\mathrm{km} \mathrm{s}^{-1}\right)\end{array}$} & \multicolumn{2}{|c|}{$\log N(\mathrm{HI})_{\mathrm{MS}}{ }^{\mathrm{c}}$} & \multicolumn{3}{|c|}{ Exposure Time (s) } & \multicolumn{2}{|c|}{$\mathrm{S} / \mathrm{N}^{\mathrm{f}}$} \\
\hline & & & & & & GASS & $\mathrm{LAB}$ & $\mathrm{UVES}^{\mathrm{d}}$ & $\mathrm{G}_{130 \mathrm{M}^{\mathrm{e}}}$ & $\mathrm{G}_{160 \mathrm{M}^{\mathrm{e}}}$ & $1300 \AA$ & $1550 \AA$ \\
\hline RBS 144 & Sey 1 & 299.485 & -65.836 & 21.6 & 92 & 20.17 & 20.27 & 6000 & 2352 & 2972 & 34 & 24 \\
\hline NGC 7714 & $\mathrm{Nuc}^{\mathrm{g}}$ & 88.216 & -55.564 & 75.9 & -320 & 19.09 & 18.93 & $\ldots h$ & 2062 & 2760 & 20 & 15 \\
\hline PHL 2525 & QSO & 80.683 & -71.317 & 60.5 & -260 & $<18.21(3 \sigma)$ & 18.24 & 5605 & 2146 & 2772 & 22 & 19 \\
\hline HE 0056-3622 & Sey1 & 293.719 & -80.898 & 36.7 & $150^{\mathrm{i}}$ & $18.70^{\mathrm{i}}$ & $18.87^{\mathrm{i}}$ & 5400 & 4598 & 5657 & 24 & 22 \\
\hline
\end{tabular}

Notes.

a Angular separation of sightline from center of SMC.

${ }^{b}$ Central LSR velocity of H I emission from the MS (or, in the case of HE 0056-3622, from the AVC).

${ }^{c}$ Logarithmic H I column density in the Stream measured from GASS (14'.4 beam) and LAB ( $30^{\prime}$ or $36^{\prime}$ beam) surveys.

d VLT/UVES exposure time with Dichroic $1390+580$ setting.

e $H S T /$ COS exposure time with G130M/1289 and G160M/1589 settings.

f Signal-to-noise ratio per resolution element of COS data at $1300 \AA$ and $1550 \AA$.

g Extended galaxy nucleus, not point-source.

${ }^{\mathrm{h}}$ No UVES data taken for this target.

i The absorption centered at $150 \mathrm{~km} \mathrm{~s}^{-1}$ toward HE 0056-3622 traces the AVCs near the south Galactic pole, not the MS.

Cloud (LMC) and Small Magellanic Cloud (SMC) stars (Olano 2004; Nidever et al. 2008). Following new measurements of the proper motion of the Magellanic Clouds (Kallivayalil et al. 2006a, 2006b, 2013; Piatek et al. 2008), which are compatible with the idea that the Magellanic Clouds are on their first passage around the Galaxy, the most recent tidal models (Besla et al. 2010, 2012; Diaz \& Bekki 2011a, 2012) confirm that the Stream is produced largely by the effect of tides raised by the LMC on the SMC during a recent encounter, as was realized earlier by Gardiner \& Noguchi (1996). The fate of the Stream has been investigated by Bland-Hawthorn et al. (2007); in their model, the MS is breaking down in a shock cascade and will subsequently rain onto the Galaxy in a warm ionized phase, or evaporate into the hot corona (see also Heitsch \& Putman 2009). Empirical determinations of the Stream's gas-phase properties are critical to test these models and thereby confirm the fate of the Stream.

With UV absorption-line studies of background quasars, a wide range of diagnostics of the physical and chemical conditions in the MS are available. Early Hubble Space Telescope (HST) measurements of the MS metallicity using the $\mathrm{S}_{\mathrm{II}} / \mathrm{H}_{\mathrm{I}}$ and $\mathrm{Si}$ II $/ \mathrm{H}$ I ratios found values $Z_{\mathrm{MS}}=0.28 \pm 0.04$ solar in the Fairall 9 direction (Gibson et al. 2000), lying close to the LMC and SMC, and $Z_{\mathrm{MS}}=0.25 \pm 0.07$ solar in the NGC 3783 direction through the Leading Arm (Lu et al. 1994, 1998; Sembach et al. 2001; Wakker et al. 2002). More recently, Fox et al. (2010, hereafter F10) reported a lower metallicity of $Z_{\mathrm{MS}}=$ $0.10 \pm 0.03$ solar in the tip of the Stream using the O I/H I ratio, which is robust against ionization and dust corrections. These measurements support the view that the Stream originates in the SMC, which has a current-day mean oxygen abundance of 0.22 solar, and not the LMC, which has a current-day mean oxygen abundance of 0.46 solar (Russell \& Dopita 1992), but until now, good statistics on the Stream's metal abundance have been lacking. In addition, caution must be used in comparing gas-phase abundances in the Stream with current-day abundances in the Clouds, since the Stream was stripped some time ago $(\approx 2$ Gyr in the tidal models), and its metallicity will reflect the metallicity of the outer regions of its parent galaxy at the time at which it was stripped, not at the present day.

We have undertaken a multi-wavelength program to study the MS using optical and UV absorption. As part of this program, we are measuring a larger set of elemental abundances in the MS for comparison with SMC and LMC abundances, thereby providing more stringent constraints on the origins of the Stream. In this paper, we present the results from UV and optical spectra of four active galactic nuclei (AGNs): RBS 144, NGC 7714, PHL 2525, and HE 0056-3622. These sightlines all lie behind the Stream, as can be seen in Figure 1, which shows a $21 \mathrm{~cm}$ emission map of the entire Magellanic region generated from the Leiden-Argentine-Bonn (LAB) survey (Kalberla et al. 2005). The sightlines sample a wide range of $\mathrm{H}$ I column density, from $\log N(\mathrm{HI})_{\mathrm{MS}}=20.17$ toward RBS 144 down to 18.24 toward PHL 2525. Basic properties of these sightlines are given in Table 1. In a companion paper, Richter et al. (2013, hereafter Paper II), we present detailed results from the Fairall 9 sightline, lying only 8.3 away on the sky from RBS 144 .

We use the term "main body" of the Stream to refer to its principal HI-emitting filaments passing from the LMC and SMC through the south Galactic pole and over into the western Galactic hemisphere (see Figure 1). The term does not refer to the Leading Arm or Interface Region of the Magellanic System, which are discussed in Brüns et al. (2005). $21 \mathrm{~cm}$ studies have shown the main body to be bifurcated both spatially (Putman et al. 2003b) and kinematically (Nidever et al. 2008, hereafter N08) into two principal filaments. N08 reported that one of the two filaments (hereafter the LMC filament) can be traced kinematically back to a star-forming area of the LMC known as the southeastern $\mathrm{H}$ I overdensity region, which contains the giant $\mathrm{H}$ II region 30 Doradus, but that the other filament (hereafter the second filament) cannot be traced to either Magellanic Cloud, so its origin until now has been unknown.

We note that RBS 144 lies behind the second filament reported by N08, whereas PHL 2525 and NGC 7714 lie near the tip of the Stream, where the filamentary structure is difficult to discern. We also note that toward HE 0056-3622, the Stream is centered near $v_{\mathrm{LSR}}=-10 \mathrm{~km} \mathrm{~s}^{-1}$, where MS absorption overlaps with foreground Galactic absorption. For this reason HE 0056-3622 is not a good probe of the MS, even though the sightline passes through it. However, the HE 0056-3622 spectrum shows highvelocity absorption at local-standard-of-rest (LSR) velocities of $80-200 \mathrm{~km} \mathrm{~s}^{-1}$ (see Section 3.5), similar to the velocities of the "anomalous velocity clouds" (AVCs) found in H i emission in directions (like HE 0056-3622) near the south Galactic pole, where the Sculptor group of galaxies is located (Mathewson et al. 1975; Haynes \& Roberts 1979; Putman et al. 2003b). 


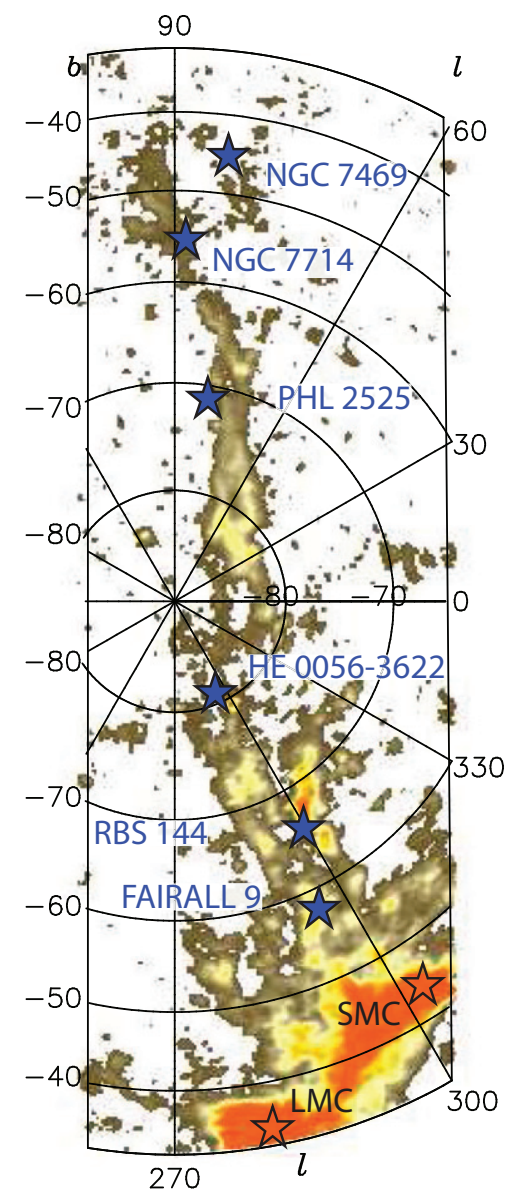

Figure 1. H i $21 \mathrm{~cm}$ map of the MS generated from the LAB survey and colorcoded by $\mathrm{H}$ I column density. The color scale ranges from $5 \times 10^{18}$ to $3 \times$ $10^{20} \mathrm{~cm}^{-2}$. The map is shown in Galactic coordinates centered on the south Galactic pole. The integration range in deviation velocity is $v_{\mathrm{dev}}=-500$ to -80 and 50 to $500 \mathrm{~km} \mathrm{~s}^{-1}$, chosen to minimize contamination by foreground emission. The four directions from this paper plus Fairall 9 (Paper II) and NGC 7469 (F10) are marked. Note how the RBS 144 and Fairall 9 directions sample the two principal filaments of the Stream.

(A color version of this figure is available in the online journal.)

HE 0056-3622 is therefore a useful probe of the AVCs. We use metallicity measurements to investigate the origin of these clouds, including the possibility that they are associated with the MS.

This paper is structured as follows. In Section 2 we describe the observations and data reduction. In Section 3 we describe the measurement techniques, and briefly discuss the observed properties of absorption in the four directions under study. In Section 4, we derive and discuss the chemical abundances in the MS, analyze the dust content of the Stream, and present Cloudy models to investigate the effects of ionization. A discussion on the implications of these results on the origin of the Stream is presented in Section 5. We summarize our findings in Section 6. Throughout this paper, we present velocities in the kinematical LSR reference frame, we take atomic data from Morton (2003), and we follow the standard definition of abundances $[\mathrm{X} / \mathrm{H}] \equiv \log (\mathrm{X} / \mathrm{H})-\log (\mathrm{X} / \mathrm{H})_{\odot}$. We adopt solar (photospheric) abundances from Asplund et al. (2009).

\section{OBSERVATIONS}

HST/COS observations of the four target AGN were taken under HST program ID 12604 (PI: A. Fox), using the G130M/
1291 and G160M/1589 settings, together giving wavelength coverage from 1132-1760 A. Details of these observations are given in Table 1. The COS instrument is described in Green et al. (2012). In all cases, the different exposures were taken at different FP-POS positions, to move the spectra on the detector and mitigate the effects of fixed-pattern noise. The CALCOS pipeline (v2.17.3) was used to process and combine the raw data, yielding a set of co-added $x 1$ dsum.fits files that were used for the analysis. No velocity offsets larger than a few $\mathrm{km} \mathrm{s}^{-1}$ were found between interstellar absorption features in these spectra, i.e., there was no evidence for significant velocity-scale calibration errors. The COS spectra have a velocity resolution $R \approx 16,000$ (instrumental FWHM $\approx 19 \mathrm{~km} \mathrm{~s}^{-1}$ ), and were rebinned by three pixels to $\approx 7.0 \mathrm{~km} \mathrm{~s}^{-1}$ pixels for display and measurement. The signal-to-noise ratios $(\mathrm{S} / \mathrm{Ns})$ of each spectra measured at $1300 \AA$ (G130M grating) and at $1550 \AA$ (G160M grating) are given in Table 1. A second, orbital nightonly reduction of the data was completed, in which the data were extracted only over those time intervals when the Sun altitude (FITS keyword SUN_ALT) was less than $20^{\circ}$. This reduction is necessary to reduce the strong geocoronal emission (airglow) in O I 1302, Si II 1304, and Ly $\alpha$ at velocities of $\left|v_{\mathrm{LSR}}\right| \lesssim 200 \mathrm{~km} \mathrm{~s}^{-1}$. Finally, the spectra were normalized around each line of interest using low-order polynomial fits to the local continuum.

Three of the four target AGN (all except NGC 7714) were observed with Very Large Telescope/Ultraviolet and Visual Echelle Spectrograph (VLT/UVES) under ESO program ID 085.C-0172(A) (PI: A. Fox). The UVES instrument is described in Dekker et al. (2000). These observations were taken in Service Mode with Dichroic 1 in the 390+580 setting, no binning, a 0 '.6 slit, and under good seeing conditions (FWHM $<0$ '.8), giving wavelength coverage from $3260-4450 \AA$ and $4760-6840 \AA$. The spectral resolution in this setting $(R \approx 70,000)$ corresponds to a velocity resolution FWHM $=4.3 \mathrm{~km} \mathrm{~s}^{-1}$. The data were reduced using the standard UVES pipeline (based on Ballester et al. 2000) running in the Common Pipeline Library environment, using calibration frames taken close in time to the corresponding science frames. The $\mathrm{S} / \mathrm{N}$ per resolution element of the UVES data at $3930 \AA$ (near Ca II) is $\approx 68$ toward RBS $144, \approx 40$ toward HE 0056-3622, and $\approx 101$ toward PHL 2525.

For our $21 \mathrm{~cm}$ analysis, we use spectra from two radio surveys, the LAB survey (Kalberla et al. 2005) and the Galactic All Sky Survey (GASS) (McClure-Griffiths et al. 2009; Kalberla et al. 2010), ${ }^{9}$ using the closest pointings to our target directions. Using measurements from two radio telescopes allows us to investigate beamsize effects, which limit the precision with which one can derive the $\mathrm{H}$ I column density (and, in turn, the metallicity) in a pencil-beam direction. The LAB survey observations were taken with either the $30 \mathrm{~m}$ Villa Elisa telescope (30' beam) or the $25 \mathrm{~m}$ Dwingeloo telescope ( $36^{\prime}$ beam). The GASS observations were taken with the $64 \mathrm{~m}$ Parkes telescope with a beam size of 14.4.

The Hi columns in the Stream were calculated from the equation $N(\mathrm{HI})=1.823 \times 10^{18} \mathrm{~cm}^{-2} \int_{v_{\min }}^{v_{\max }} T_{b} d v$ (e.g., Dickey \& Lockman 1990), where $T_{b}$ is the brightness temperature in Kelvin, $v_{\min }$ and $v_{\max }$ are the velocity integration limits in $\mathrm{km} \mathrm{s}^{-1}$, and the line is assumed to be optically thin. The differences in the H I columns derived from the LAB and GASS spectra are visible in Table 1. We adopt these differences as the beamsize uncertainty in the H I column density in the MS

\footnotetext{
9 Data from both surveys are available at http://www.astro.uni-bonn.de/hisurvey/profile.
} 
along the line-of-sight to each AGN. This uncertainty translates directly to the derived metal abundances, and we include it as a systematic error in our results, although toward RBS 144 we are able to confirm the MS H I column in the pencil-beam line-ofsight using a fit to the damping wings of $\operatorname{Ly} \alpha$ (see Section 3.2).

\section{MAGELLANIC STREAM ABSORPTION}

Figure 2 shows the VLT/UVES data covering Ca II, Na I, and Ti II for the RBS 144, PHL 2525, and HE 0056-3622 directions. Figures 3-6 show the HST/COS data for the same three directions plus NGC 7714, for which no UVES data are available. In each case we include the $21 \mathrm{~cm}$ emission-line profile for comparison, from either the GASS or LAB survey. The $21 \mathrm{~cm}$ data are shown unbinned in Figure 2, but for display are rebinned to five pixels in Figures 3-6. We now discuss the measurement techniques and present an overview of the MS (or AVC) absorption seen in each direction.

\subsection{Measurements}

We used the apparent optical depth (AOD) technique of Savage \& Sembach (1991) to measure the absorption in the LSR velocity range of the MS for each metal line of interest in the COS and UVES data sets. In this technique, the AOD in each pixel as calculated as $\tau_{a}(v)=\ln \left[F_{c}(v) / F(v)\right]$, where $F_{c}$ is the continuum level and $F$ is the flux. The apparent column density in each pixel is then given by $N_{a}(v)=\left(m_{e} c / \pi e^{2}\right)\left[\tau_{a}(v) / f \lambda\right]=$ $3.768 \times 10^{14}\left[\tau_{a}(v) / f \lambda\right] \mathrm{cm}^{-2}\left(\mathrm{~km} \mathrm{~s}^{-1}\right)^{-1}$, where $f$ is the oscillator strength of the transition and $\lambda$ is the wavelength in Angstroms. This can be integrated over the profile to give the apparent column density, $N_{a}=\int_{v_{\min }}^{v_{\max }} N_{a}(v) d v$. The apparent column densities in the MS derived from this technique are given in Tables 2-4 for the RBS 144, NGC 7714, and PHL 2525 directions. The apparent column densities in the AVC toward HE 0056-3622 are given in Table 5.

All our data have sufficient $\mathrm{S} / \mathrm{N}$ (see Table 1 ) to lie in the regime where the AOD method is reliable (Fox et al. 2005a), so long as no unresolved saturation is present. This could occur if narrow unresolved lines are present in the profiles, which is more likely for the COS data than the UVES data. In cases where doublets or multiplets of the same ion are present in the data (e.g., Si II), we used these to check for saturation. When lines are not detected at $3 \sigma$ significance, we give upper limits on $N_{a}$ based on the noise measured in the continuum.

For the UVES data, Voigt-component fits to the optical (Ca II, Ti II, and Na I, where present) lines were used to determine the low-ion component structure in the Stream, using the VPFIT software package. ${ }^{10}$ These fits account for the UVES instrumental line spread function, assumed to be a Gaussian with a FWHM of $4.3 \mathrm{~km} \mathrm{~s}^{-1}$. Each ion was fit independently, with the number of components chosen to be the minimum necessary to match the data (see Figure 2): three MS components for RBS 144 and two AVC components for HE 0056-3622. Note that we use the UVES data to derive the component structure because of their superior velocity resolution compared to the COS data $\left(4.3 \mathrm{~km} \mathrm{~s}^{-1}\right.$ versus $\approx 19 \mathrm{~km} \mathrm{~s}^{-1}$ FWHM), though we note that $\mathrm{Ca}$ II does not necessarily trace the same low-ion phase as the UV metal lines because of its low ionization potential.

\subsection{MS Absorption toward RBS 144}

The central LSR velocity of the MS in the RBS 144 direction is $92 \mathrm{~km} \mathrm{~s}^{-1}$, as defined by the peak of the GASS $21 \mathrm{~cm}$ emission

\footnotetext{
10 Available at http://www.ast.cam.ac.uk/ rfc/vpfit.html.
}

Table 2

MS Column Densities and Ion Abundances toward RBS144

\begin{tabular}{|c|c|c|c|c|c|}
\hline Ion & $\begin{array}{l}\text { Line } \\
(\AA)\end{array}$ & $\log (\mathrm{X} / \mathrm{H})_{\odot}^{\mathrm{a}}$ & $\begin{array}{l}\mathrm{IP}^{\mathrm{b}} \\
(\mathrm{eV})\end{array}$ & $\begin{array}{l}\log N_{a}(\mathrm{MS}) \\
\left(N_{a} \text { in } \mathrm{cm}^{-2}\right)\end{array}$ & {$\left[\mathrm{X}^{i} / \mathrm{H}\right]_{\mathrm{MS}}^{\mathrm{c}}$} \\
\hline $\mathrm{HI}_{\mathrm{I}}$ & $21 \mathrm{~cm}$ & 0.0 & 13.6 & $20.17^{\mathrm{d}}$ & $\ldots$ \\
\hline $\mathrm{OI}_{\mathrm{I}}$ & 1302 & -3.31 & 13.6 & $>14.69$ & $>-2.16$ \\
\hline $\mathrm{C}_{\mathrm{I}}$ & 1656 & -3.57 & 11.3 & $<13.39$ & $<-3.20$ \\
\hline $\mathrm{C}_{\text {II }}$ & 1334 & -3.57 & 24.4 & $>14.62$ & $>-1.98$ \\
\hline Al II & 1670 & -5.55 & 18.8 & $12.86 \pm 0.05^{\mathrm{e}}$ & $-1.75 \pm 0.05^{\mathrm{e}}$ \\
\hline Ni II & 1370 & -5.78 & 18.2 & $<13.84$ & $<-0.54$ \\
\hline Si II & 1260 & -4.49 & 16.3 & $>13.68$ & $>-1.99$ \\
\hline Si II & 1193 & -4.49 & 16.3 & $>13.88$ & $>-1.80$ \\
\hline Si II & 1190 & -4.49 & 16.3 & $>13.99$ & $>-1.68$ \\
\hline Si II & 1526 & -4.49 & 16.3 & $14.12 \pm 0.04$ & $-1.56 \pm 0.04$ \\
\hline Si II & 1304 & -4.49 & 16.3 & $14.09 \pm 0.09$ & $-1.58 \pm 0.09$ \\
\hline Si III & 1206 & -4.49 & 33.5 & $>13.56$ & $>-2.12$ \\
\hline$S_{\text {II }}$ & 1259 & -4.88 & 23.3 & $14.16 \pm 0.12^{\mathrm{f}}$ & $-1.13 \pm 0.12^{\mathrm{f}}$ \\
\hline $\mathrm{S}_{\text {II }}$ & 1253 & -4.88 & 23.3 & $<14.49$ & $<-0.79$ \\
\hline$S_{\text {II }}$ & 1250 & -4.88 & 23.3 & $<14.82$ & $<-0.46$ \\
\hline Fe II & 1608 & -4.50 & 16.2 & $14.12 \pm 0.08$ & $-1.55 \pm 0.08$ \\
\hline Fe II & 1144 & -4.50 & 16.2 & $13.99 \pm 0.07$ & $-1.68 \pm 0.07$ \\
\hline $\mathrm{N}_{\mathrm{I}}$ & 1200.0 & -4.17 & 14.5 & $<14.10$ & $<-1.90$ \\
\hline C IV & 1548 & -3.57 & 64.5 & $<13.51$ & $<-3.08$ \\
\hline C IV & 1550 & -3.57 & 64.5 & $<13.86$ & $<-2.74$ \\
\hline Si IV & 1393 & -4.49 & 45.1 & $<13.11$ & $<-2.57$ \\
\hline Si IV & 1402 & -4.49 & 45.1 & $<13.25$ & $<-2.43$ \\
\hline $\mathrm{Nv}$ & 1238 & -4.17 & 97.9 & $<13.48$ & $<-2.51$ \\
\hline $\mathrm{Nv}$ & 1242 & -4.17 & 97.9 & $<13.66$ & $<-2.33$ \\
\hline Ca II & 3934 & -5.66 & 11.9 & $12.27 \pm 0.02$ & $-2.23 \pm 0.10$ \\
\hline Ca II & 3969 & -5.66 & 11.9 & $12.34 \pm 0.03$ & $-2.16 \pm 0.10$ \\
\hline $\mathrm{NaI}$ & 5891 & -5.76 & 5.1 & $<11.12$ & $<-3.28$ \\
\hline $\mathrm{NaI}$ & 5897 & -5.76 & 5.1 & $<11.42$ & $<-2.99$ \\
\hline Ti II & 3384 & -7.05 & 13.6 & $<11.81$ & $<-1.30$ \\
\hline
\end{tabular}

Notes. LSR velocity integration range for MS in this direction is $65-210 \mathrm{~km} \mathrm{~s}^{-1}$ For O I 1302 and Si II 1304, measurements were made on night-only data.

a Solar photospheric abundance (Asplund et al. 2009).

b Ionization potential $X^{i} \rightarrow X^{i+1}$.

${ }^{\mathrm{c}}\left[\mathrm{X}^{i} / \mathrm{H}\right]_{\mathrm{MS}}=\log \left[\mathrm{X}^{i} / \mathrm{H} \mathrm{I}\right]_{\mathrm{MS}}-\log (\mathrm{X} / \mathrm{H})_{\odot}$. Upper/lower limits are $3 \sigma / 1 \sigma$. These abundances are not corrected for ionization. Only the statistical error is given.

$\mathrm{d}$ Value given is GASS survey measurement (14.4 beam). LAB survey gives $\log N(\mathrm{HI})=20.27\left(30^{\prime}\right.$ beam $)$.

e Line potentially saturated.

f Velocity range adjusted to $65-135 \mathrm{~km} \mathrm{~s}^{-1}$ to avoid blend.

profile. The Hi profile extends over the range $75-140 \mathrm{~km} \mathrm{~s}^{-1}$. The UV absorption lines show absorption centered near the same velocity, but covering a more extended interval of $65-210 \mathrm{~km} \mathrm{~s}^{-1}$ (Figure 3).

The following UV metal lines are detected in the MS: O I 1302, C II 1334, Si II 1260, 1193, 1190, 1526, 1304, Si III 1206, S II 1259, Al II 1670, and Fe II 1144, 1608. Several of these lines are saturated; the lines which appear unsaturated, from which we derive ionic column densities, are Si II 1526, 1304, S II 1259, Al II 1670, and Fe II 1144, 1608 (see Table 2). However, for Al II 1670, we cannot rule out unresolved saturation, since the line reaches a normalized depth of 0.8 . No detection is seen in the high ion doublets C IV 1548, 1550, Si IV 1393, 1402, and Nv 1238, 1242, or in the low-ion lines Ni II 1370, S II 1253, 1250, or N I 1200.7. Among the N I 1199.5, 1200.2, 1200.7 triplet, only the 1200.7 line is shown on Figure 3 since absorption at MS velocities in the first two lines is blended. Two high-velocity components can be discerned in the UV profiles. The primary MS component (closely aligned with the $21 \mathrm{~cm}$ emission) is centered near $100 \mathrm{~km} \mathrm{~s}^{-1}$. A secondary component 
Table 3

MS Column Densities and Ion Abundances toward NGC 7714

\begin{tabular}{|c|c|c|c|c|c|}
\hline Ion & $\begin{array}{l}\text { Line } \\
(\AA)\end{array}$ & $\log (\mathrm{X} / \mathrm{H})_{\odot}^{\mathrm{a}}$ & $\begin{array}{l}\mathrm{IP}^{\mathrm{b}} \\
(\mathrm{eV})\end{array}$ & $\begin{array}{c}\log N_{a}(\mathrm{MS}) \\
\left(N_{a} \text { in } \mathrm{cm}^{-2}\right)\end{array}$ & {$\left[\mathrm{X}^{i} / \mathrm{H}\right]_{\mathrm{MS}}{ }^{\mathrm{c}}$} \\
\hline $\mathrm{H} \mathrm{I}$ & $21 \mathrm{~cm}$ & 0.0 & 13.6 & $19.09^{\mathrm{d}}$ & 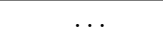 \\
\hline $\mathrm{O}_{\mathrm{I}}$ & 1302 & -3.31 & 13.6 & $14.54 \pm 0.12$ & $-1.24 \pm 0.12$ \\
\hline $\mathrm{C}_{\mathrm{I}}$ & 1656 & -3.57 & 11.3 & $<13.75$ & $<-1.77$ \\
\hline $\mathrm{C}_{\text {II }}$ & 1334 & -3.57 & 24.4 & $14.47 \pm 0.10$ & $-1.05 \pm 0.10$ \\
\hline $\mathrm{Al}$ II & 1670 & -5.55 & 18.8 & $<12.76$ & $<-0.78$ \\
\hline Ni II & 1370 & -5.78 & 18.2 & $<14.06$ & $<0.75$ \\
\hline Si II & 1193 & -4.49 & 16.3 & $13.66 \pm 0.11$ & $-0.94 \pm 0.11$ \\
\hline Si II & 1190 & -4.49 & 16.3 & $13.95 \pm 0.11$ & $-0.65 \pm 0.11$ \\
\hline Si II & 1526 & -4.49 & 16.3 & $13.94 \pm 0.12$ & $-0.66 \pm 0.12$ \\
\hline Si II & 1304 & -4.49 & 16.3 & $<14.09$ & $<-0.51$ \\
\hline Si III & 1206 & -4.49 & 33.5 & $13.67 \pm 0.10$ & $-0.93 \pm 0.10$ \\
\hline S II & 1253 & -4.88 & 23.3 & $<14.95$ & $<0.74$ \\
\hline Fe II & 1608 & -4.50 & 16.2 & $<14.23$ & $<-0.36$ \\
\hline Fe II & 1144 & -4.50 & 16.2 & $<13.98$ & $<-0.61$ \\
\hline CIV & 1548 & -3.57 & 64.5 & $13.93 \pm 0.12$ & $-1.59 \pm 0.12$ \\
\hline CIV & 1550 & -3.57 & 64.5 & $13.99 \pm 0.14$ & $-1.53 \pm 0.14$ \\
\hline Si IV & 1393 & -4.49 & 45.1 & $13.52 \pm 0.11$ & $-1.08 \pm 0.11$ \\
\hline Si IV & 1402 & -4.49 & 45.1 & $<13.70$ & $<-0.90$ \\
\hline $\mathrm{Nv}$ & 1238 & -4.17 & 97.9 & $<13.64$ & $<-1.28$ \\
\hline
\end{tabular}

Notes. LSR velocity integration range for MS in this direction is -420 to $-190 \mathrm{~km} \mathrm{~s}^{-1}$. For O I 1302 and Si II 1304, measurements were made on nightonly data.

a Solar photospheric abundance (Asplund et al. 2009).

b Ionization potential $X^{i} \rightarrow X^{i+1}$.

${ }^{c}\left[\mathrm{X}^{i} / \mathrm{H}\right]_{\mathrm{MS}}=\log \left[\mathrm{X}^{i} / \mathrm{HI}\right]_{\mathrm{MS}}-\log (\mathrm{X} / \mathrm{H})_{\odot}$. Upper/lower limits are $3 \sigma / 1 \sigma$. These abundances are not corrected for ionization. Only the statistical error is given.

d Value given is GASS survey measurement (14.'4 beam).

Table 4

MS Column Densities and Ion Abundances toward PHL2525

\begin{tabular}{|c|c|c|c|c|c|}
\hline Ion & $\begin{array}{l}\text { Line } \\
(\AA)\end{array}$ & $\log (\mathrm{X} / \mathrm{H})_{\odot}^{\mathrm{a}}$ & $\begin{array}{l}\mathrm{IP}^{\mathrm{b}} \\
(\mathrm{eV})\end{array}$ & $\begin{array}{l}\log N_{a}(\mathrm{MS}) \\
\left(N_{a} \text { in } \mathrm{cm}^{-2}\right)\end{array}$ & {$\left[\mathrm{X}^{i} / \mathrm{H}\right]_{\mathrm{MS}}^{\mathrm{c}}$} \\
\hline $\mathrm{HI}$ & $21 \mathrm{~cm}$ & 0.0 & 13.6 & $18.24^{\mathrm{d}}$ & $\ldots$ \\
\hline $\mathrm{O}_{\mathrm{I}}$ & 1302 & -3.31 & 13.6 & $<14.30$ & $<-0.63$ \\
\hline $\mathrm{C}_{\mathrm{I}}$ & 1656 & -3.57 & 11.3 & $<13.69$ & $<-0.98$ \\
\hline $\mathrm{C}_{\text {II }}$ & 1334 & -3.57 & 24.4 & $14.38 \pm 0.04$ & $-0.29 \pm 0.04$ \\
\hline Al II & 1670 & -5.55 & 18.8 & $<12.58$ & $<-0.11$ \\
\hline Ni II & 1370 & -5.78 & 18.2 & $<14.02$ & $<1.56$ \\
\hline Si II & 1193 & -4.49 & 16.3 & $13.38 \pm 0.08$ & $-0.37 \pm 0.08$ \\
\hline Si II & 1190 & -4.49 & 16.3 & $13.61 \pm 0.09$ & $-0.14 \pm 0.09$ \\
\hline Si II & 1526 & -4.49 & 16.3 & $<13.75$ & $<-0.00$ \\
\hline Si II & 1304 & -4.49 & 16.3 & $<13.92$ & $<0.17$ \\
\hline Si III & 1206 & -4.49 & 33.5 & $>13.70$ & $>-0.05$ \\
\hline S II & 1259 & -4.88 & 23.3 & $<14.54$ & $<1.18$ \\
\hline S II & 1253 & -4.88 & 23.3 & $<14.73$ & $<1.37$ \\
\hline Fe II & 1608 & -4.50 & 16.2 & $<14.11$ & $<0.37$ \\
\hline Fe II & 1144 & -4.50 & 16.2 & $<14.05$ & $<0.30$ \\
\hline Civ & 1548 & -3.57 & 64.5 & $13.99 \pm 0.05$ & $-0.68 \pm 0.05$ \\
\hline CiV & 1550 & -3.57 & 64.5 & $14.02 \pm 0.09$ & $-0.65 \pm 0.09$ \\
\hline Si IV & 1393 & -4.49 & 45.1 & $13.20 \pm 0.10$ & $-0.55 \pm 0.10$ \\
\hline $\mathrm{Nv}$ & 1238 & -4.17 & 97.9 & $<13.56$ & $<-0.52$ \\
\hline $\mathrm{Nv}$ & 1242 & -4.17 & 97.9 & $<13.77$ & $<-0.30$ \\
\hline
\end{tabular}

Notes. LSR velocity integration range for MS in this direction is -280 to $-120 \mathrm{~km} \mathrm{~s}^{-1}$. For O I 1302 and Si II 1304, measurements were made on nightonly data.

a Solar photospheric abundance (Asplund et al. 2009).

b Ionization potential $X^{i} \rightarrow X^{i+1}$.

${ }^{c}\left[\mathrm{X}^{i} / \mathrm{H}\right]_{\mathrm{MS}}=\log \left[\mathrm{X}^{i} / \mathrm{H} \mathrm{I}\right]_{\mathrm{MS}}-\log (\mathrm{X} / \mathrm{H})_{\odot}$. Upper/lower limits are $3 \sigma / 1 \sigma$. These abundances are not corrected for ionization. Only the statistical error is given.

d Value given is GASS survey measurement (14.4 beam).
Table 5

AVC Column Densities and Ion Abundances toward HE0056-3622

\begin{tabular}{|c|c|c|c|c|c|}
\hline Ion & $\begin{array}{l}\text { Line } \\
(\AA)\end{array}$ & $\log (\mathrm{X} / \mathrm{H})_{\odot}^{\mathrm{a}}$ & $\begin{array}{l}\mathrm{IP}^{\mathrm{b}} \\
(\mathrm{eV})\end{array}$ & $\begin{array}{c}\log N_{a}(\mathrm{MS}) \\
\left(N_{a} \text { in } \mathrm{cm}^{-2}\right)\end{array}$ & {$\left[\mathrm{X}^{i} / \mathrm{H}\right]_{\mathrm{MS}}{ }^{\mathrm{c}}$} \\
\hline $\mathrm{HI}_{\mathrm{I}}$ & $21 \mathrm{~cm}$ & 0.0 & 13.6 & $18.70^{\mathrm{d}}$ & $\ldots$ \\
\hline $\mathrm{OI}_{\mathrm{I}}$ & 1302 & -3.31 & 13.6 & $14.36 \pm 0.07$ & $-1.03 \pm 0.07$ \\
\hline $\mathrm{C}_{\mathrm{I}}$ & 1656 & -3.57 & 11.3 & $<13.54$ & $<-1.59$ \\
\hline $\mathrm{C}_{\text {II }}$ & 1334 & -3.57 & 24.4 & $>14.38$ & $>-0.75$ \\
\hline Al II & 1670 & -5.55 & 18.8 & $12.54 \pm 0.08$ & $-0.61 \pm 0.08$ \\
\hline Ni II & 1370 & -5.78 & 18.2 & $<13.64$ & $<0.72$ \\
\hline Si II & 1260 & -4.49 & 16.3 & $>13.36$ & $>-0.85$ \\
\hline Si II & 1193 & -4.49 & 16.3 & $>13.58$ & $>-0.63$ \\
\hline Si II & 1190 & -4.49 & 16.3 & $13.70 \pm 0.04$ & $-0.51 \pm 0.04$ \\
\hline Si II & 1526 & -4.49 & 16.3 & $13.77 \pm 0.05$ & $-0.44 \pm 0.05$ \\
\hline Si II & 1304 & -4.49 & 16.3 & $13.78 \pm 0.11$ & $-0.42 \pm 0.11$ \\
\hline Si III & 1206 & -4.49 & 33.5 & $>13.39$ & $>-0.82$ \\
\hline S II & 1253 & -4.88 & 23.3 & $<14.37$ & $<0.55$ \\
\hline S II & 1250 & -4.88 & 23.3 & $<14.74$ & $<0.92$ \\
\hline Fe II & 1608 & -4.50 & 16.2 & $<13.97$ & $<-0.23$ \\
\hline Fe II & 1144 & -4.50 & 16.2 & $<13.73$ & $<-0.47$ \\
\hline $\mathrm{N}_{\mathrm{I}}$ & 1200.0 & -4.17 & 14.5 & $<14.11$ & $<-0.42$ \\
\hline Civ & 1548 & -3.57 & 64.5 & $<13.42$ & $<-1.71$ \\
\hline CIV & 1550 & -3.57 & 64.5 & $<13.48$ & $<-1.65$ \\
\hline Si IV & 1393 & -4.49 & 45.1 & $<12.81$ & $<-1.40$ \\
\hline $\mathrm{N} \mathrm{V}$ & 1238 & -4.17 & 97.9 & $<13.22$ & $<-1.31$ \\
\hline $\mathrm{N} \mathrm{v}$ & 1242 & -4.17 & 97.9 & $<13.66$ & $<-0.86$ \\
\hline Ca II & 3934 & -5.66 & 11.9 & $11.99 \pm 0.04$ & $-1.05 \pm 0.11$ \\
\hline Ca II & 3969 & -5.66 & 11.9 & $12.13 \pm 0.06$ & $-0.91 \pm 0.12$ \\
\hline $\mathrm{Na} I$ & 5891 & -5.76 & 5.1 & $<11.37$ & $<-1.57$ \\
\hline $\mathrm{Na} I$ & 5897 & -5.76 & 5.1 & $<11.63$ & $<-1.31$ \\
\hline Ti II & 3384 & -7.05 & 13.6 & $<11.94$ & $<0.30$ \\
\hline
\end{tabular}

Notes. LSR velocity integration range for AVC in this direction is 80-200 $\mathrm{km} \mathrm{s}^{-1}$. For O I 1302 and Si II 1304, measurements were made on night-only data.

${ }^{a}$ Solar photospheric abundance (Asplund et al. 2009).

${ }^{\mathrm{b}}$ Ionization potential $X^{i} \rightarrow X^{i+1}$.

${ }^{c}\left[\mathrm{X}^{i} / \mathrm{H}\right]_{\mathrm{MS}}=\log \left[\mathrm{X}^{i} / \mathrm{HI}\right]_{\mathrm{MS}}-\log (\mathrm{X} / \mathrm{H})_{\odot}$. Upper/lower limits are $3 \sigma / 1 \sigma$. These abundances are not corrected for ionization. Only the statistical error is given.

d Value given is GASS survey measurement (14.4 beam). LAB survey gives $\log N(\mathrm{Hi})=18.87\left(30^{\prime}\right.$ beam $)$.

at $180 \mathrm{~km} \mathrm{~s}^{-1}$ is seen in C II, Si II, and Si III, but not in O I or S II, or H i $21 \mathrm{~cm}$ (Figure 3).

The VLT/UVES data give MS detections of Ca II 3934, 3969 and Ti II 3384 (low significance), and upper limits on $\mathrm{Na}$ I 5891, 5897. Three components of MS absorption are visible in the Ca II profile, spread over $25 \mathrm{~km} \mathrm{~s}^{-1}$, a much narrower interval than the UV metal lines, with narrow $b$-values of $6.1 \pm 0.4 \mathrm{~km} \mathrm{~s}^{-1}, 3.0 \pm 1.6 \mathrm{~km} \mathrm{~s}^{-1}$, and $6.2 \pm 0.5 \mathrm{~km} \mathrm{~s}^{-1}$. These lines trace the cool-gas component structure in the Stream (see Paper II). An offset of $6 \mathrm{~km} \mathrm{~s}^{-1}$ is seen between the velocity centroids of the $\mathrm{HI}$ and Ca II MS components (see Figure 2): the $\mathrm{H}$ I MS emission is centered at $92 \mathrm{~km} \mathrm{~s}^{-1}$ (in both the LAB and the GASS data), whereas the Ca II absorption is centered at $98 \pm$ $1 \mathrm{~km} \mathrm{~s}^{-1}$. Furthermore, the two higher-velocity components seen in Ca II at $107 \pm 1 \mathrm{~km} \mathrm{~s}^{-1}$ and $120 \pm 1 \mathrm{~km} \mathrm{~s}^{-1}$ show no analogs in the $21 \mathrm{~cm}$ data. This indicates that small-scale structure exists in the pencil-beam sightline that is not resolved in the $21 \mathrm{~cm}$ beam.

However, since the HI column in the MS in this direction is high enough to contribute to the damping wings on the Galactic Ly $\alpha$ line, we can derive $N(\mathrm{HI})_{\mathrm{MS}}$ in the pencilbeam line-of-sight by fitting the observed Ly $\alpha$ profile with a 

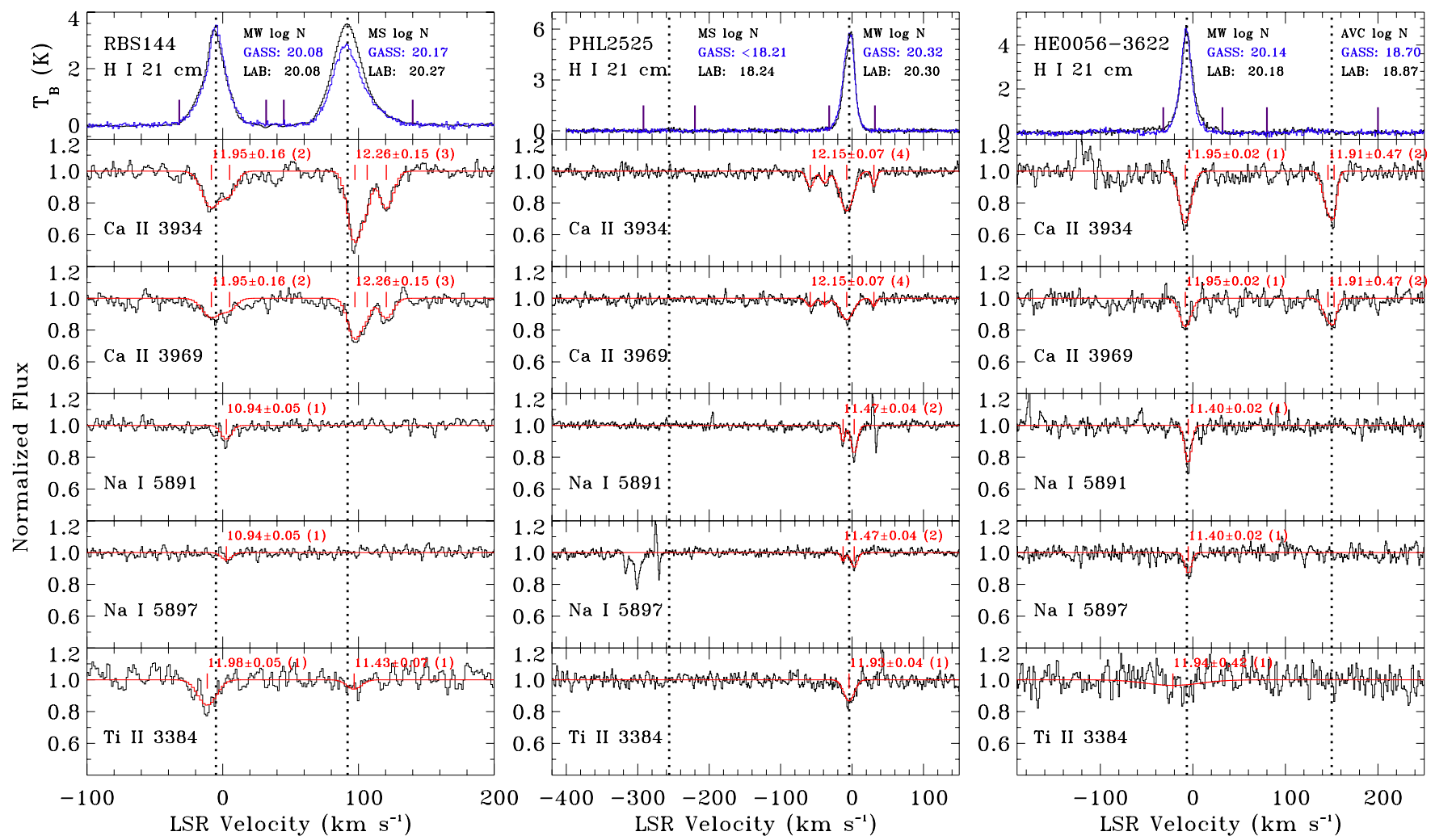

Figure 2. VLT/UVES spectra of RBS 144, PHL 2525, and HE 0556-3622. The top panels show the $21 \mathrm{~cm} \mathrm{H}$ i spectra from the LAB (black) and GASS (blue) surveys. All other panels show the UVES spectra in black, and our Voigt-profile fits convolved with the line spread function in red. The total column densities in the MW, MS, and/or AVC components are annotated above the spectra, with the number of velocity components given in parentheses. Red tick marks show the centroids of each velocity component. We fit the Ti II MS absorption toward RBS 144 with a single component (bottom-left panel), but it is not a $3 \sigma$ detection, so we treat is as an upper limit in the analysis. The vertical dotted lines show the velocity centroids of the MW and MS $21 \mathrm{~cm}$ emission components. Note the compressed $y$-scale on each panel.

(A color version of this figure is available in the online journal.)

two-component (MW+MS) model, following Lehner et al. (2008). This is important since it eliminates the systematic error on metallicities that derives from comparing UV lines measured with an infinitesimal beam with $21 \mathrm{~cm} \mathrm{H}$ I measurements made with a finite beam. We fix the velocity components at $-5 \pm$ $5 \mathrm{~km} \mathrm{~s}^{-1}$ (MW component) and $95 \pm 5 \mathrm{~km} \mathrm{~s}^{-1}$ (MS component) to follow the component structure seen in the UV metal lines, and allow only the H I column density in each component to vary. Using a fourth-order polynomial fit to the continuum, we find the $\operatorname{Ly} \alpha$ profile is well reproduced $\left(\chi_{v}^{2}=0.88\right)$ by a model with $\log N(\mathrm{HI})_{\mathrm{MW}}=20.00 \pm 0.05$ and $\log N\left(\mathrm{HI}_{\mathrm{MS}}=\right.$ $20.09 \pm 0.04$ (see Figure 7). The latter column matches the GASS value $\log N(\mathrm{HI})_{\mathrm{MS}}=20.17$ within $2 \sigma$, illustrating that in this direction the systematic uncertainty on $\log N(\mathrm{HI})_{\mathrm{MS}}$ due to beamsize effects is small $(\lesssim 0.1 \mathrm{dex})$. The accuracy of this technique is limited by the true component structure; if additional components are present, the relative columns in the MW and MS components would change. Further discussion of these limitations is given in Lehner et al. (2008). This technique cannot be used to derive $N(\mathrm{H} \mathrm{I})_{\mathrm{MS}}$ along the other three sightlines in this paper since in those directions $N(\mathrm{HI})_{\mathrm{MW}} \gg N(\mathrm{HI})_{\mathrm{MS}}$.

\subsection{MS Absorption toward NGC 7714}

NGC 7714 is an On-Stream direction showing a well-defined $21 \mathrm{~cm}$ emission component at $-320 \mathrm{~km} \mathrm{~s}^{-1}$ with $\log N(\mathrm{H} \mathrm{I})_{\mathrm{MS}}=$ 19.09 measured from the GASS data (18.93 measured from the LAB data), and UV absorption centered at the same velocity but extending over a wider interval of -420 to $-190 \mathrm{~km} \mathrm{~s}^{-1}$ (Figure 4). The UV lines detected in the MS are O I 1302,
C II 1334, Si II 1193, 1190, 1526, Si III 1206 and the high-ion lines C IV 1548, 1550 and Si IV 1393. The component structure in the UV lines is fairly simple, with a single broad component well aligned with the $21 \mathrm{~cm}$ emission. Since this target is slightly extended (a starburst nucleus rather than a point source), the absorption is not as well defined as for the other sightlines in our sample. No UVES data are available for this sightline. Ionic column densities are given in Table 3.

\subsection{MS Absorption toward PHL 2525}

The LAB $21 \mathrm{~cm}$ data toward PHL 2525 (36' beam) show a very weak emission component $\log N(\mathrm{HI})_{\mathrm{MS}}=18.24$ at $-260 \mathrm{~km} \mathrm{~s}^{-1}$ (see Figure 5, top-left panel). We identify this component with the MS. This component is not visible in the GASS data (14.4 beam), which give $\log N\left(\mathrm{HI}_{\mathrm{MS}}<18.21\right.$ $(3 \sigma)$, marginally inconsistent with the LAB data. UV absorption covering the interval -300 to $-100 \mathrm{~km} \mathrm{~s}^{-1}$ is seen in C II 1334, Si II 1193, 1190, Si III 1206, CiV 1548, 1550, and Si IV 1393, 1402 (Figure 5). No absorption in this interval is seen in the UVES data covering Ca II, Na I, and Ti II (Figure 2). There is a clear offset of $\approx 50 \mathrm{~km} \mathrm{~s}^{-1}$ between the center of the UV absorption and the LAB H I emission, further indicating the beamsize mismatch. Because of this issue, this sightline is of little use for deriving robust abundances. However, the combination of the non-detection of O I 1302 in the MS velocity interval with the strength of the LAB H I emission allows us to place an upper limit on the MS metallicity (see Section 4). Ionic column densities for this sightline are given in Table 4. 


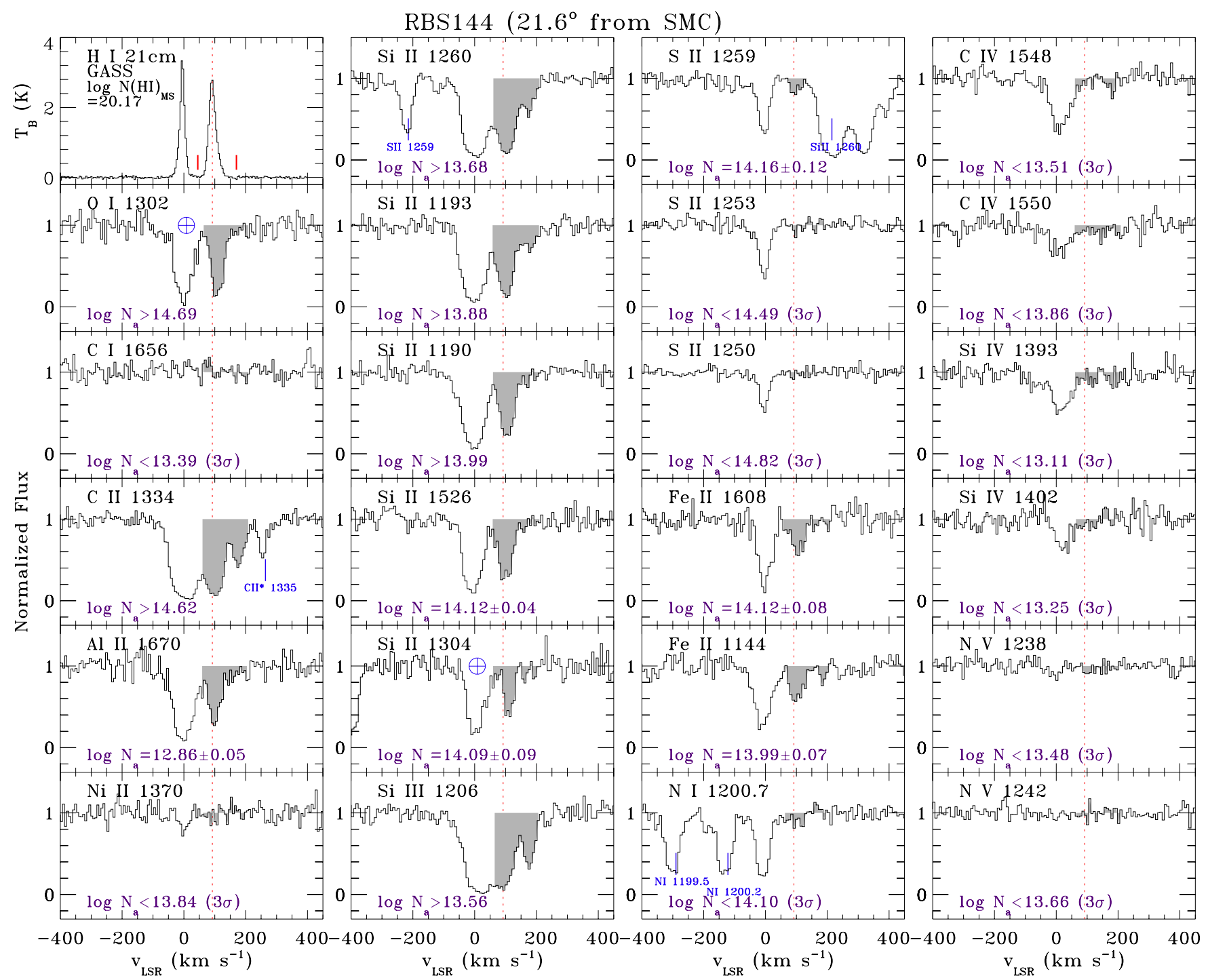

Figure 3. HST/COS metal-line profiles in the UV spectrum of RBS 144, plus the H $21 \mathrm{~cm}$ profile from the GASS survey. Normalized flux is plotted against LSR velocity for each absorption line. Gray shading indicates the MS absorption velocity interval $\left(65-210 \mathrm{~km} \mathrm{~s}^{-1}\right)$, and the vertical dotted red line shows the central velocity of the MS $21 \mathrm{~cm}$ emission. MW absorption in each panel is visible near $0 \mathrm{~km} \mathrm{~s}^{-1}$. Blends are indicated at the appropriate velocity with a tick mark and accompanying label. The apparent column density of MS absorption is indicated in the lower corner of each panel, with upper limits given for non-detections. For O I 1302 and Si II 1304, night-only data are shown to minimize the geocoronal emission.

(A color version of this figure is available in the online journal.)

\subsection{AVC Absorption toward HE 0056-3622}

In this direction high-velocity absorption is detected in many UV lines centered at $150 \mathrm{~km} \mathrm{~s}^{-1}$ and covering the interval $80-200 \mathrm{~km} \mathrm{~s}^{-1}$ (Figure 6). As discussed in Section 1, this absorption appears to trace the AVCs found near the south Galactic pole (Putman et al. 2003b). These AVCs may have an origin with the Sculptor group of galaxies (Mathewson et al. 1975), whose velocities are low as $70 \mathrm{~km} \mathrm{~s}^{-1}$, or may represent fragments of the MS (Haynes \& Roberts 1979) that have separated kinematically from the principal filaments. The $21 \mathrm{~cm}$ emission component from the AVC is weak, with $\log N(\mathrm{HI})_{\mathrm{AVC}}=$ 18.70 measured over the interval $80-200 \mathrm{~km} \mathrm{~s}^{-1}$ in the GASS data. The AVC is detected in absorption in O I 1302, C II 1334, Si II 1260, 1193, 1190, 1526, 1304, Si III 1206, Al II 1670, and Ca II 3934, 3969, but not in S II 1253, 1250, Fe II 1144, 1608, C IV 1548, 1550, Si Iv 1393, 1402, Na I 5891, 5897, or Ti II 3384. Among the detected lines, O I 1302, Al II 1670, Si II 1190, 1526,
1304, and Ca II 3934, 3969 are unsaturated and can be used to determine the ionic column densities (Table 5).

We fit two closely-spaced components to the Ca II absorption centered near $150 \mathrm{~km} \mathrm{~s}^{-1}$ (Figure 2), but the Ca II absorption does not cover the full velocity interval of the AVC absorption seen in the UV lines, which show one component centered at $100 \mathrm{~km} \mathrm{~s}^{-1}$, and a second weaker component seen in Si III 1206 and Si II 1260 at $170 \mathrm{~km} \mathrm{~s}^{-1}$.

\section{CHEMICAL ABUNDANCES IN THE MS}

The UV data contain a rich variety of diagnostics on elemental abundances in the Stream. Among the available abundance indicators, the $\mathrm{OI} / \mathrm{HI}_{\mathrm{I}}$ and $\mathrm{S}_{\mathrm{II}} / \mathrm{H}_{\mathrm{I}}$ ratios are considered the most reliable, being the least affected by dust and ionization effects (Savage \& Sembach 1996; Meyer et al. 1998; Jensen et al. 2005; Jenkins 2009). However, at the high H I columns $[\log N(\mathrm{HI}) \approx 20]$ and low metallicities $(\mathrm{O} / \mathrm{H} \approx 0.1$ solar $)$ found 


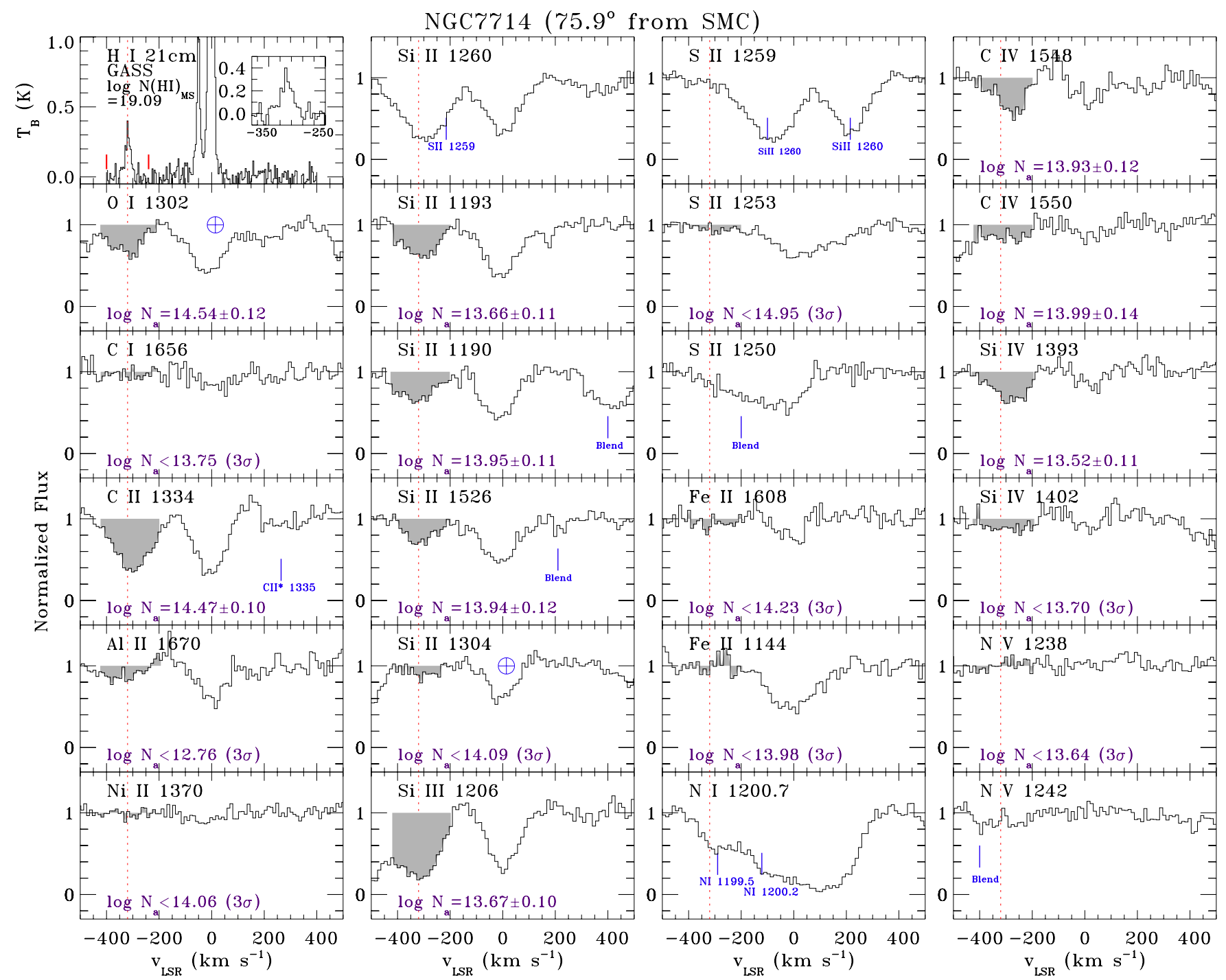

Figure 4. Same as Figure 3, for the NGC 7714 direction. Gray shading indicates MS absorption ( -420 to $\left.-190 \mathrm{~km} \mathrm{~s}^{-1}\right)$, and the vertical dotted red line shows the central velocity of the MS $21 \mathrm{~cm}$ emission. The inset in the $21 \mathrm{~cm}$ panel shows a zoom-in around the MS component.

(A color version of this figure is available in the online journal.)

in much of the MS, O I 1302 saturates yet O I 1356 is too weak to detect, so only a lower limit on the O I column is measurable. In this regime, $\mathrm{S}$ II/H I is the better metallicity indicator. Thus the metallicity indicator we use in each sightline depends on the $\mathrm{H} \mathrm{I}$ column.

Toward RBS 144, we measure $\log N_{\mathrm{a}}(\mathrm{O} \mathrm{I})>14.69$ from the strongly saturated O I 1302 line, giving a non-constraining limit $\left[\mathrm{O}_{\mathrm{I}} / \mathrm{HI}\right]_{\mathrm{MS}}>-2.17$. Turning to $\mathrm{S}_{\mathrm{II}} / \mathrm{HI}$, we use the $\mathrm{S}_{\mathrm{II}}$ column measured from an AOD integration of S II 1259 to derive $[\mathrm{S} \text { II } / \mathrm{HI}]_{\mathrm{MS}}=-1.13 \pm 0.12$ (stat) \pm 0.10 (syst), where the first error is the statistical uncertainty and the second error represents the difference between the LAB and GASS H I column densities and accounts for the beamsize mismatch between the radio and UV observations (see F10 for a more detailed discussion of the size of this systematic error). Adding the two errors in quadrature to give an overall uncertainty gives $[\mathrm{S} \mathrm{II} / \mathrm{HI}]_{\mathrm{MS}}=-1.13 \pm$ 0.16 . The $\mathrm{S}$ II 1259 line is only detected at $3.3 \sigma$ significance in the co-added spectrum (Figure 3), but is seen in both individual sub-exposures. A non-detection would serve to lower the derived metallicity. For this S II 1259 line only, we adjusted the velocity integration range to $65-135 \mathrm{~km} \mathrm{~s}^{-1}$ instead of $65-210 \mathrm{~km} \mathrm{~s}^{-1}$, to avoid blending with Galactic Si II 1260 . Fortunately the $21 \mathrm{~cm}$
MS signal is almost all contained in this restricted velocity range, so the $\mathrm{S}$ abundance is not affected by this adjustment. Using the non-detection of MS absorption in N I 1200.7, we derive $\log N\left(\mathrm{NI}_{\mathrm{I}}\right)<14.10$ and $\left[\mathrm{N} \mathrm{I}_{\mathrm{I}} \mathrm{HI}_{\mathrm{I}}\right]_{\mathrm{MS}}<-1.90(3 \sigma)$. Nitrogen is therefore underabundant in the gas phase of the Stream, with $\left[\mathrm{N} / \mathrm{S}_{\mathrm{MS}}=\left[\mathrm{N}_{\mathrm{I}} / \mathrm{S}_{\mathrm{II}}\right]_{\mathrm{MS}}<-0.77(3 \sigma)\right.$.

Toward NGC 7714, we measure $\log N_{\mathrm{a}}(\mathrm{O} \mathrm{I})=14.54 \pm 0.06$ in the MS from the night-only O I 1302 data, corresponding to $[\mathrm{O} \mathrm{I} / \mathrm{HI}]_{\mathrm{MS}}=-1.24 \pm 0.12$ (stat) \pm 0.16 (syst) $=-1.24 \pm 0.20$ when combining the errors in quadrature. Toward PHL 2525, we measure $\log N_{\mathrm{a}}(\mathrm{O} \mathrm{I})<14.30(3 \sigma)$ in the MS, again from nightonly O I 1302 data, corresponding to $[\mathrm{O} \mathrm{I} / \mathrm{HI}]_{\mathrm{MS}}<-0.63(3 \sigma)$. Toward HE 0056-3622, we measure $\log N_{\mathrm{a}}(\mathrm{O} \mathrm{I})=14.36 \pm 0.07$ in the AVC from the night-only O I 1302 data, corresponding to $[\mathrm{O} \mathrm{I} / \mathrm{H} \mathrm{I}]_{\mathrm{AVC}}=-1.03 \pm 0.07$ (stat) \pm 0.17 (syst) $=-1.03 \pm 0.18$. The OI 1302 line in the AVC appears unsaturated (Figure 6), with a maximum normalized depth of 0.7 , although we cannot rule out the possibility of unresolved saturation; if present, this would raise the value of $\mathrm{O} / \mathrm{H}$ in the AVC. An upper limit on the $\mathrm{S}$ II abundance measured from the non-detection of $\mathrm{S}$ II 1253 toward HE 0056-3622 is [S II $\left./ \mathrm{HI}_{\mathrm{I}}\right]_{\mathrm{AVC}}<+0.55(3 \sigma)$, consistent with the $\mathrm{O}$ I/H I abundance, but not constraining. 


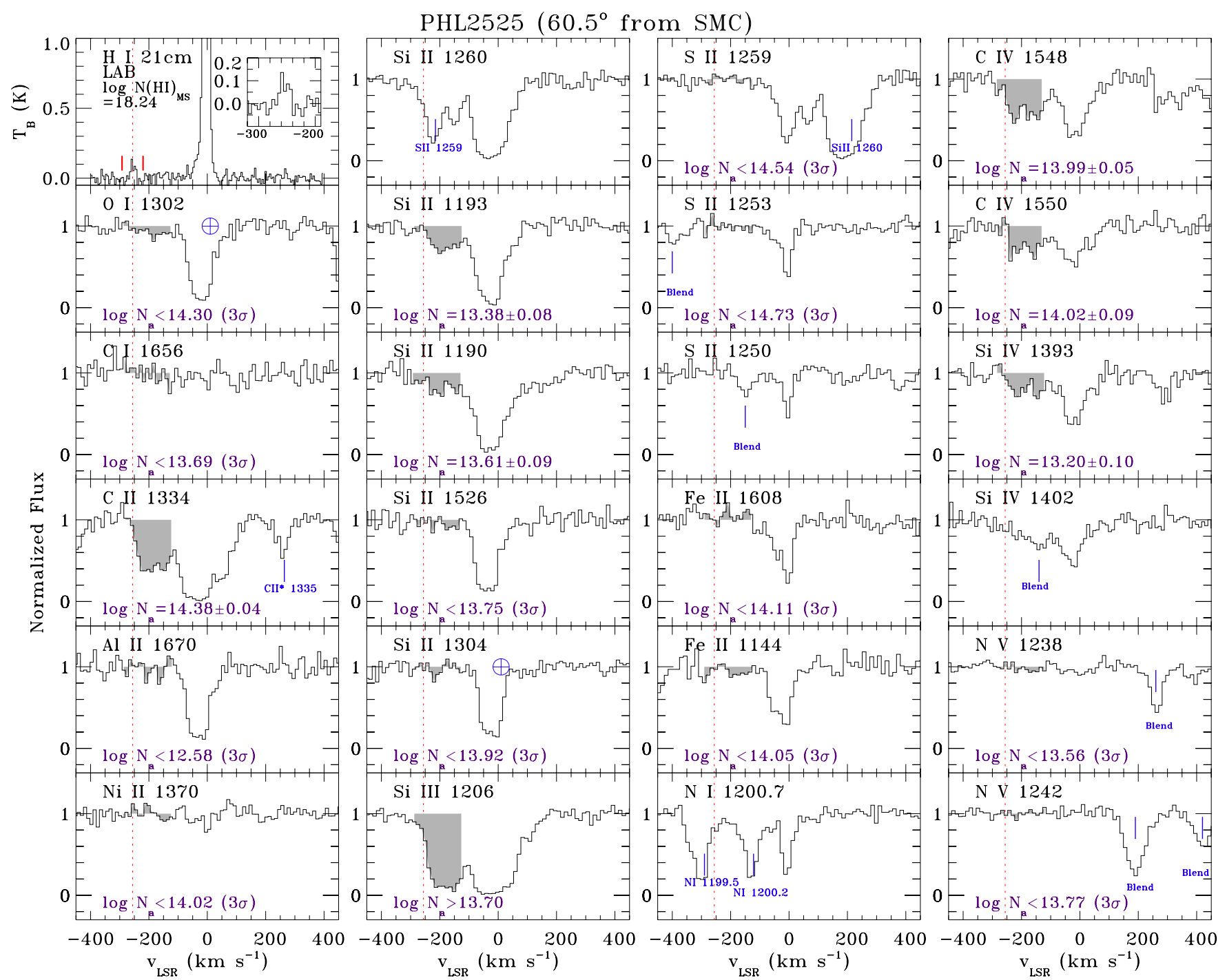

Figure 5. Same as Figure 3, for the PHL 2525 direction. Gray shading indicates MS absorption $\left(-280\right.$ to $\left.-120 \mathrm{~km} \mathrm{~s}^{-1}\right)$, and the vertical dotted red line shows the central velocity of the (weak) MS $21 \mathrm{~cm}$ emission. No O I 1302 absorption is detected in the MS so only an upper limit on $[\mathrm{O} / \mathrm{H}]_{\mathrm{MS}}$ can be derived.

(A color version of this figure is available in the online journal.)

\subsection{Ion Abundances}

In this sub-section we focus on the low-ion species and their relative proportion with H I. These ratios form empirical indicators of the gas-phase abundances. They are listed in Tables 2-5 and are plotted in Figure 8 for ten low-ionization species, in order of increasing atomic number.

The top panel of Figure 8 compares the MS ion abundances measured toward RBS 144 (this paper; $\log N(\mathrm{HI})_{\mathrm{MS}}=20.17$ ) and Fairall 9 (Paper II; $\left.\log N(\mathrm{HI})_{\mathrm{MS}}=19.95\right)$ with the compilation of LMC and SMC reference abundances presented by Russell \& Dopita (1992), which includes abundances measured in stars and in interstellar emission-line objects such as supernova remnants and $\mathrm{H}$ II regions. Comparing the RBS 144 and Fairall 9 data points, a clear pattern is seen, in which the ion abundances (or limits) are lower toward RBS 144 than toward Fairall 9 for all ions shown. This indicates that as the H I column goes down, the low-ion metal columns do not decrease in linear proportion. The MS ion abundances measured toward RBS 144 are consistently lower than the current-day elemental abundances in the LMC and SMC. The difference is smallest for sulfur, which shows a 0.6 dex difference between the MS and the current-day SMC value. The Magellanic Cloud abundances vary substantially from one element to another, which complicates the interpretation of the MS abundance pattern.

In the lower panel of Figure 8, we compare the dust depletions of each element measured in the MS toward RBS 144 and Fairall 9 with those measured in the Magellanic Bridge (MB) by Lehner et al. (2001) and Lehner (2002), and to the compilation of MW halo depletions presented by Welty et al. (1997), which was based on Savage \& Sembach (1996) and Fitzpatrick (1996). The Bridge measurements are made in the direction of O-star DI 1388, whereas the halo cloud measurements represent the averages over a sample of many sightlines. We define the depletion of element $\mathrm{X}$ relative to sulfur as $\delta(\mathrm{X}) \equiv[\mathrm{X} / \mathrm{S} \mathrm{II}]=[\mathrm{X} / \mathrm{H}]-[\mathrm{S} \mathrm{II} / \mathrm{H}]$. The depletions measured in the two MS sightlines are generally similar, with a notable discrepancy for Ca II: $\delta(\mathrm{Ca})$ is $0.44 \mathrm{dex}$ lower toward Fairall 9 than toward RBS 144. The Bridge and Stream show a depletion pattern that is consistent for all ions shown, particularly for $\delta(\mathrm{Fe})$ and $\delta(\mathrm{Si})$, where values rather than limits are available. The MS dust depletion pattern toward 


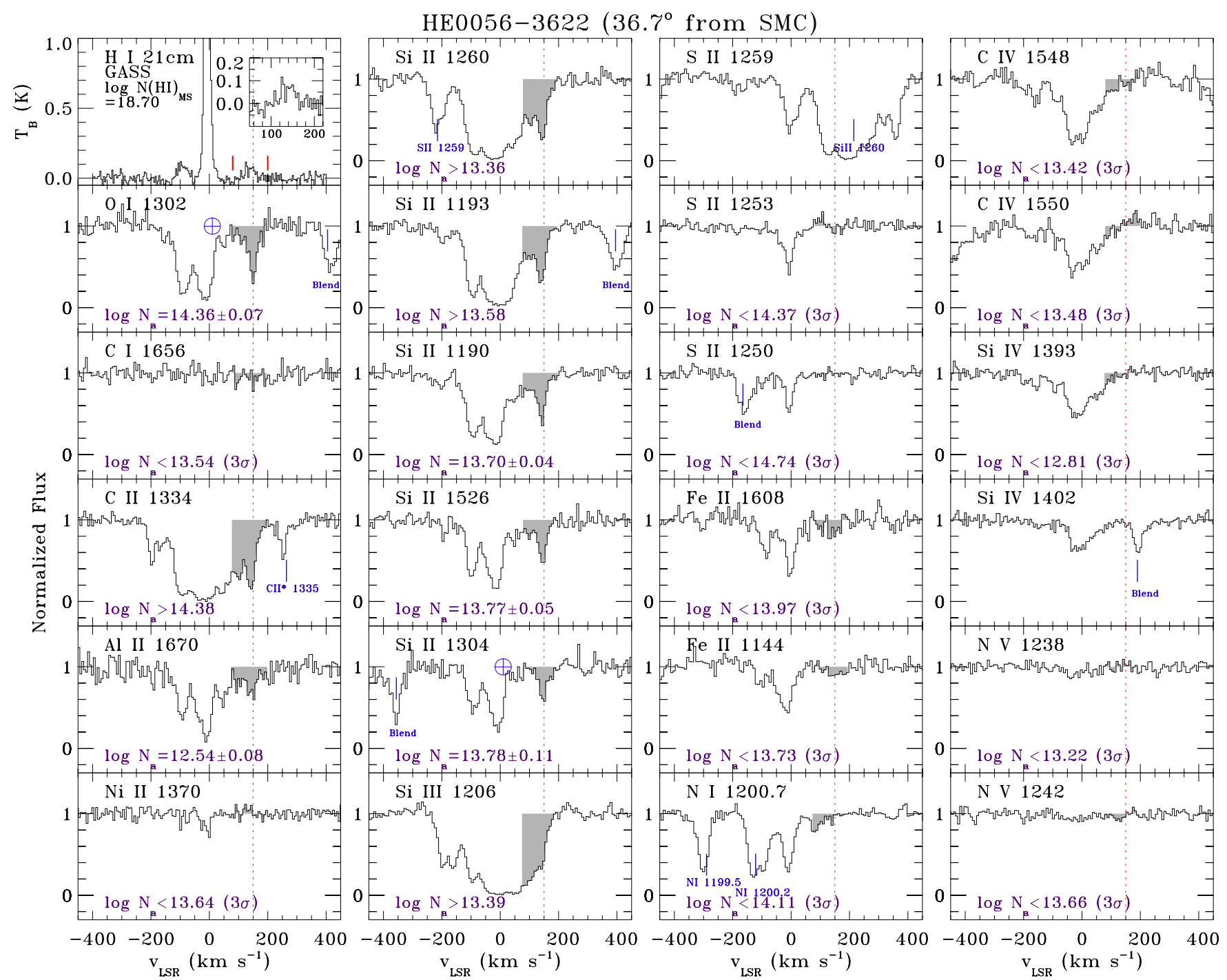

Figure 6. Same as Figure 3, for the HE 0056-3622 direction. Gray shading indicates anomalous-velocity cloud (AVC) absorption (80-200 km s ${ }^{-1}$ ). MS absorption in this direction is centered near $-10 \mathrm{~km} \mathrm{~s}^{-1}$ and overlaps with Galactic foreground absorption. The inset in the $21 \mathrm{~cm}$ panel shows a zoom-in around the AVC component.

(A color version of this figure is available in the online journal.)

RBS 144 is also consistent with the Galactic halo depletion pattern for all elements shown except nitrogen, which is underabundant in the MS in this direction relative to the Galactic halo by $\gtrsim 0.7$ dex (although the halo value is highly uncertain).

\subsection{Gas-to-dust Ratios}

A useful number to derive when discussing the chemical properties of the Stream is the gas-to-dust mass ratio, which is directly related to the depletion of $\mathrm{Fe}$ atoms onto dust grains. We define this ratio following Wolfe et al. (2003) as $\mathrm{G} / \mathrm{D} \equiv N(\mathrm{H}) /$ $N(\mathrm{Fe})_{\mathrm{d}}$ where $N(\mathrm{Fe})_{d}=N(\mathrm{~S})(\mathrm{Fe} / \mathrm{S})_{\odot}\left(1-10^{[\mathrm{Fe} / \mathrm{S}]}\right)$ is the column of iron in dust grains. This assumes that a solar $\mathrm{Fe} / \mathrm{S}$ ratio applies to the entire (dust+gas) system. The single sightline in our sample with both Fe II and S II detections (and hence for which we can calculate G/D) is RBS 144; in this direction, we derive $(\mathrm{G} / \mathrm{D})_{\mathrm{MS}}=600000_{-190000}^{+32000}$. We can normalize this to the Galactic ISM value by writing $(\mathrm{G} / \mathrm{D})_{\text {norm }}=(\mathrm{G} / \mathrm{D})_{\mathrm{MS}} /$ $(\mathrm{G} / \mathrm{D})_{\mathrm{MW}}$. Since $(\mathrm{G} / \mathrm{D})_{\mathrm{MW}} \approx(\mathrm{Fe} / \mathrm{H})_{\odot}^{-1}$ as Fe is almost entirely depleted in the Galactic ISM, we find $(\mathrm{G} / \mathrm{D})_{\text {norm }}=19_{-6}^{+10}$. Along the nearby Fairall 9 sightline discussed in Paper II, we find a lower ratio $(\mathrm{G} / \mathrm{D})_{\mathrm{MS}}=104000_{-14000}^{+17000}$ and $(\mathrm{G} / \mathrm{D})_{\text {norm }}=3.3_{-0.5}^{+0.5}$. This corresponds to a higher dust content toward Fairall 9, as expected given the higher MS metallicity in that direction.

For comparison, measurements of $(\mathrm{G} / \mathrm{D})_{\text {norm }}$ in the SMC lie in the range $\approx 5-14$ (Sofia et al. 2006; Leroy et al. 2007; Gordon et al. 2009; J. Roman-Duval et al. 2013, in preparation), whereas the LMC has a lower average ratio $(\mathrm{G} / \mathrm{D})_{\text {norm }} \approx 2-4$ (Draine 2003). The ratio observed in the MS toward RBS 144 is therefore closer to (though higher than) the current-day ratio observed in the diffuse gas of the SMC, whereas the ratio measured toward Fairall 9 is equal to the LMC value.

\subsection{Ionization Corrections}

Up to now we have considered ion abundances, not elemental abundances. The abundance of the species $\mathrm{O}$ I and $\mathrm{S}$ II are robust indicators of the elemental abundances of $\mathrm{O}$ and $\mathrm{Si}$, as discussed above, but for other species, ionization corrections must be applied to derive the elemental abundances. These corrections can be derived under the standard assumption that the lowionization species (singly and doubly ionized) are photoionized 

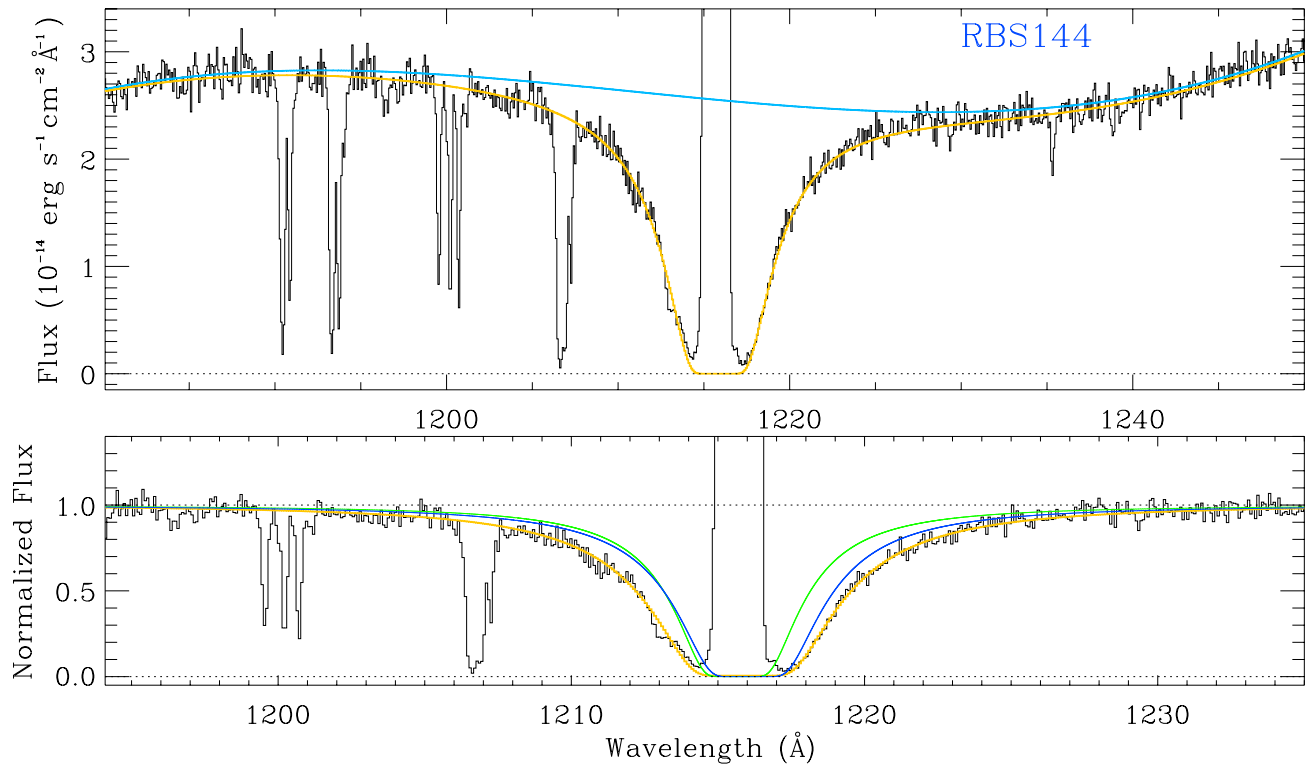

Figure 7. Derivation of $N(\mathrm{HI}$ ) toward RBS 144 from a two-component (MW+MS) fit to the damping wings of Ly $\alpha$ (shown in yellow), using a fourth-order polynomial fit to the continuum (shown in blue). The upper and lower panels show the raw and normalized profiles, respectively. The strong emission feature near the center of each panel is geocoronal Ly $\alpha$ emission. The fit yields $\log N(\mathrm{HI})_{\mathrm{MW}}=20.00 \pm 0.05$ and $\log N(\mathrm{HI})_{\mathrm{MS}}=20.09 \pm 0.04$, which are in reasonable agreement with the columns derived from the GASS $21 \mathrm{~cm}$ data. The contributions from the two components are shown in the lower panel as green (MW) and blue (MS) curves. These COS data have been binned to the instrumental resolution of $18 \mathrm{~km} \mathrm{~s}^{-1}$.

(A color version of this figure is available in the online journal.)
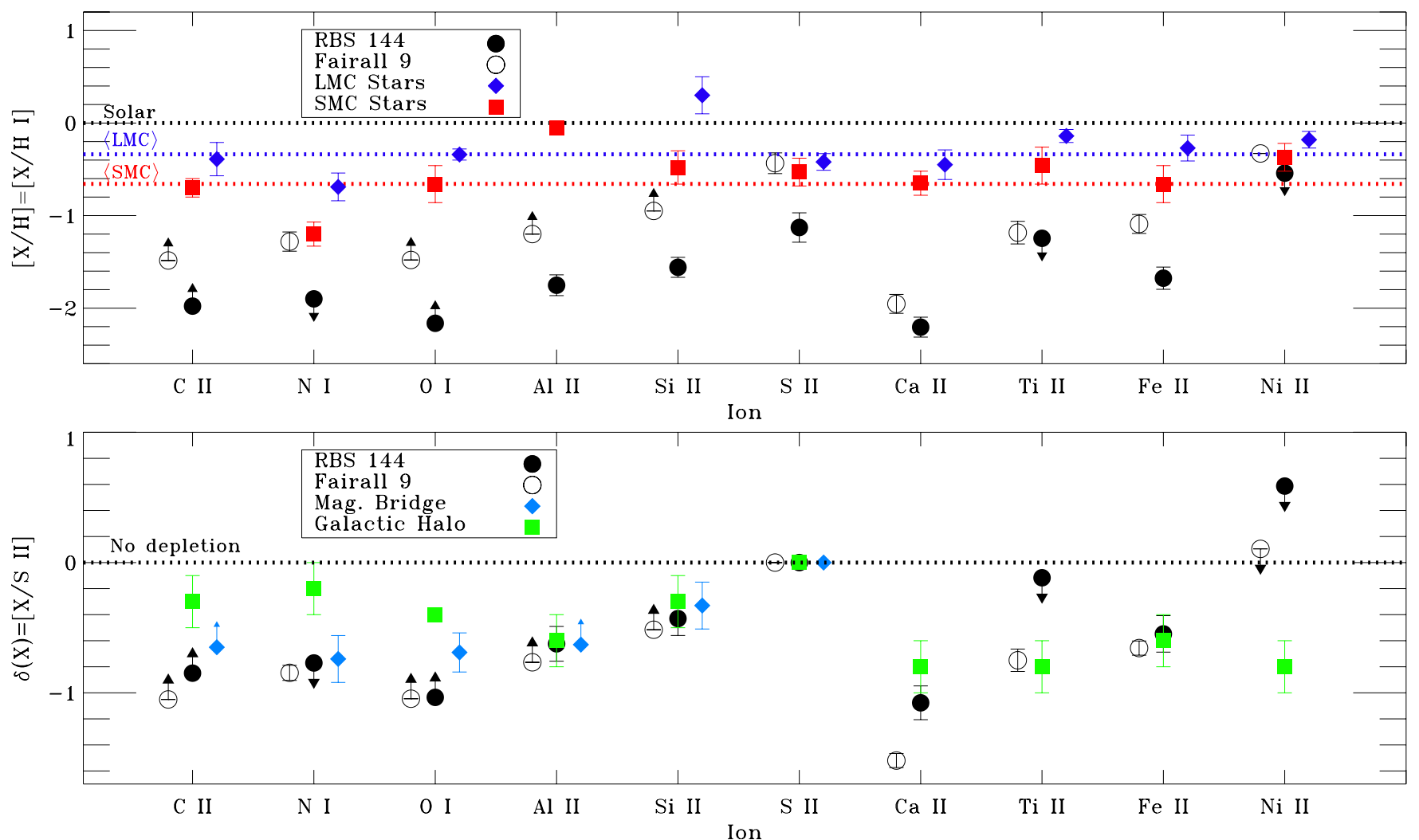

Figure 8. Upper panel: comparison of MS ion abundances measured toward RBS 144 (this paper) and Fairall 9 (Paper II) with the SMC and LMC abundance patterns presented by Russell \& Dopita (1992). The solar, average LMC (0.46 solar), and average SMC (0.22 solar) abundances are plotted as horizontal lines. Consistently lower ion abundances are seen toward RBS $144\left[\log N(\mathrm{HI})_{\mathrm{MS}}=20.17\right]$ than toward Fairall $9\left[\log N(\mathrm{HI})_{\mathrm{MS}}=19.95\right]$. Lower panel: comparison of the dust depletion levels $\delta(\mathrm{X})=[\mathrm{X} / \mathrm{S}$ II $]$ measured in the MS toward RBS 144 and Fairall 9 with the dust depletion pattern measured in the Magellanic Bridge by Lehner (2002), and with the Galactic halo depletion compilation of Welty et al. (1997). All values are uncorrected for ionization. The data points have been offset in the $x$-direction for clarity. See Section 4.1 for discussion.

(A color version of this figure is available in the online journal.) 
by the incident radiation field. We ran a set of photoionization models using Cloudy v10.00 (Ferland et al. 1998) to investigate this process, assuming the gas exists in a plane-parallel slab of uniform density. We follow the two-step method outlined in F10. First, we solve for the value of the ionization parameter $\log U \equiv \log \left(n_{\gamma} / n_{\mathrm{H}}\right)$ necessary to produce the observed $\mathrm{Si}$ III/Si II ratio, where $n_{\gamma}$ is the density of $\mathrm{H}$-ionizing photons $(\lambda<912 \AA)$. Second, we solve for the abundances of all the low-ion elements by comparing their observed columns with those predicted by the model.

This method does not assume a priori that the heavy element abundances are in their solar ratios; instead it solves for the abundance of each element separately, assuming the low-ions arise in the same gas phase as the H I. See Nigra et al. (2012) for a discussion of effects occurring if clumpiness changes this assumption. The models combine the UV background radiation calculated by Haardt \& Madau (2012) with a model of the escaping ionizing MW radiation from Fox et al. (2005b), which was based on Bland-Hawthorn \& Maloney $(1999,2002)$. The MW radiation field is non-isotropic, with the escape fraction highest normal to the Galactic disk, so $n_{\gamma}$ changes with latitude (Fox et al. 2005b).

We ran Cloudy models for two of the four sightlines in this paper, RBS 144 (chosen to model the MS since it has the most UV metal lines detected) and HE 0056-3622 (to model the AVCs near the south Galactic pole). For the RBS 144 sightline, we use a model with $l, b, R=299.5,-65.8,50 \mathrm{kpc}$ appropriate if the Stream is at the same distance as the Magellanic Clouds, which gives $\log \left(n_{\gamma} / \mathrm{cm}^{-3}\right)=-5.61$. For the HE 0056-3622 sightline, we adopt $l, b, R=293.7,-80.9,50 \mathrm{kpc}$ giving $\log \left(n_{\gamma} / \mathrm{cm}^{-3}\right)=-5.34$. We do not include the radiation escaping from the Magellanic Clouds or the radiation produced by the shock cascade or interface regions within the Stream. The inclusion of additional radiation fields would increase the photon density, so that for a given ionization parameter the clouds would become denser and smaller.

The results of our Cloudy models are given in two sets of figures. First, Figure 9 shows the ionization corrections for six low ions calculated from the Cloudy models, as a function of $\log U$, for the RBS 144 and HE 0056-3622 directions. We also include a model appropriate to the Fairall 9 sightline, for use in the discussion and for comparison to Paper II. The ionization corrections are defined as the difference between the intrinsic elemental abundance and the measured ion abundance, i.e., $\mathrm{IC}\left(\mathrm{X}^{\mathrm{i}}\right)=[\mathrm{X} / \mathrm{H}]-\left[\mathrm{X}^{\mathrm{i}} / \mathrm{H} \mathrm{I}\right]$. Second, Figures 10(a) (for RBS 144) and 10(b) (for HE 0056-3622) show the columns of the observed low ions as a function of $\log U$, with the derived abundances of each element and the best-fit $\log U$ annotated on the panel. The results drawn from these two sets of figures are discussed in the next two sub-sections.

\subsubsection{Cloudy Results toward RBS 144}

We measure a ratio $\log [N(\mathrm{Si}$ III 1206) $/ N(\mathrm{Si}$ II 1526) $] \gtrsim-0.56$ over the full MS velocity interval $65-210 \mathrm{~km} \mathrm{~s}^{-1}$. Reproducing this ratio with a Cloudy model yields an ionization parameter $\log U \gtrsim-3.2$, corresponding to a gas density $\log \left(n_{\mathrm{H}} / \mathrm{cm}^{-3}\right) \lesssim-2.4$ (see Figure $\left.10(\mathrm{a})\right)$. These are limits since the MS component in Si III 1206 is saturated. This model has an ionized gas column $\log N(\mathrm{H} \mathrm{II})=19.9$. If we repeat the measurement in the velocity interval 150-200 $\mathrm{km} \mathrm{s}^{-1}$, where Si III 1206 and $\mathrm{Si}$ II 1260 show a single, unsaturated component, we derive $\log [N($ Si III 1206) $/ N($ Si II 1260) $]=-0.13$. Reproducing this ratio yields a best-fit $\log U \gtrsim-2.8$ and $\log \left(n_{\mathrm{H}} / \mathrm{cm}^{-3}\right) \lesssim-2.8$,
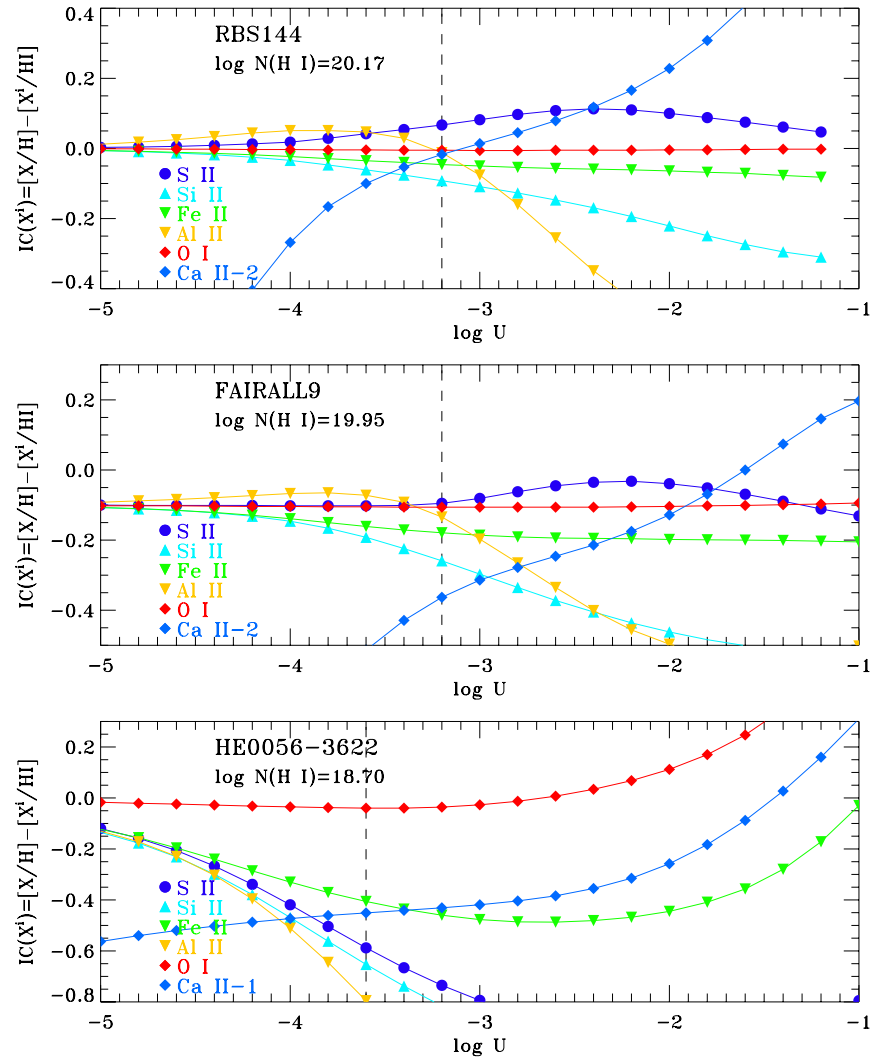

Figure 9. Ionization corrections for six low ions (O I, S II, Si II, Al II, Fe II, and Ca II) in our Cloudy models of the MS toward RBS 144 (top) and Fairall 9 (center), and of the AVCs toward HE 0056-3622 (bottom). These corrections show the amount which must be added to the observed ion-to-H I ratio to determine the intrinsic abundance. For RBS 144 and Fairall 9, the corrections for all ions shown are $\lesssim 0.10 \mathrm{dex}$ at the best-fit value for $\log U$ (derived by matching the Si III/Si II ratios), indicating that the sub-solar values derived for $\mathrm{Si} / \mathrm{S}, \mathrm{Al} / \mathrm{S}$, and $\mathrm{Fe} / \mathrm{S}$ in the MS can be attributed to dust depletion, not ionization. For HE 0056-3622, the models support the use of $\mathrm{O}$ I/H I as a robust abundance indicator even in the regime $\log N(\mathrm{HI})<19$, at least for $\log U \lesssim-2.0$. Note that the Ca II curves have been offset by -1.0 or -2.0 dex on the $y$-axis for comparison to the other ions.

(A color version of this figure is available in the online journal.)

so there is a $\approx 0.4$ dex uncertainty in the best-fit density that results from saturation effects.

Regardless of the difficulty in determining the density to high precision, the simulations confirm that the $[\mathrm{S} \mathrm{II} / \mathrm{HI}$ ] ratio is a good indicator of the overall $\mathrm{S}$ abundance $[\mathrm{S} / \mathrm{H}]$ in the MS: in our best-fit model along this sightline at $\log U \gtrsim-3.2$, the ionization correction is found to be $\mathrm{IC}(\mathrm{S}$ II $)=0.07 \mathrm{dex}$, and the correction is $<0.1$ dex over four orders of magnitude in density (Figure 9, top panel). Physically, this is because the fraction of $\mathrm{S}$ in the form of $\mathrm{S}^{+2}$ or higher is similar to the fraction of $\mathrm{H}$ in the form of $\mathrm{H}^{+}$(see Lu et al. 1998; Howk et al. 2006; Howk \& Consiglio 2012, for related discussions).

The simulations confirm that $\mathrm{Al}, \mathrm{Si}$, and $\mathrm{Fe}$ are underabundant with respect to $S$ in the MS, as we found in Figure 8 based on the ion abundances alone. As for $\mathrm{S}$, the ionization corrections for Al II, Si II, and Fe II derived by the Cloudy model at $\log U=$ -3.2 are small: $\mathrm{IC}(\mathrm{Al} \mathrm{II})=-0.01 \mathrm{dex}, \mathrm{IC}(\mathrm{Si} \mathrm{II})=-0.09 \mathrm{dex}$, and $\mathrm{IC}(\mathrm{Fe}$ II $)=-0.05 \mathrm{dex}$, which is a consequence of the high H I column in the Stream in this direction. Applying these corrections we derive gas-phase abundances $[\mathrm{Al} / \mathrm{H}]_{\mathrm{MS}}=-1.8$, $[\mathrm{Si} / \mathrm{H}]_{\mathrm{MS}}=-1.7$, and $[\mathrm{Fe} / \mathrm{H}]_{\mathrm{MS}}=-1.7$, corresponding to moderate dust depletion levels $\delta(\mathrm{Al})_{\mathrm{MS}} \approx-0.7, \delta(\mathrm{Si})_{\mathrm{MS}} \approx$ -0.6 , and $\delta(\mathrm{Fe})_{\mathrm{MS}} \approx-0.6$. 

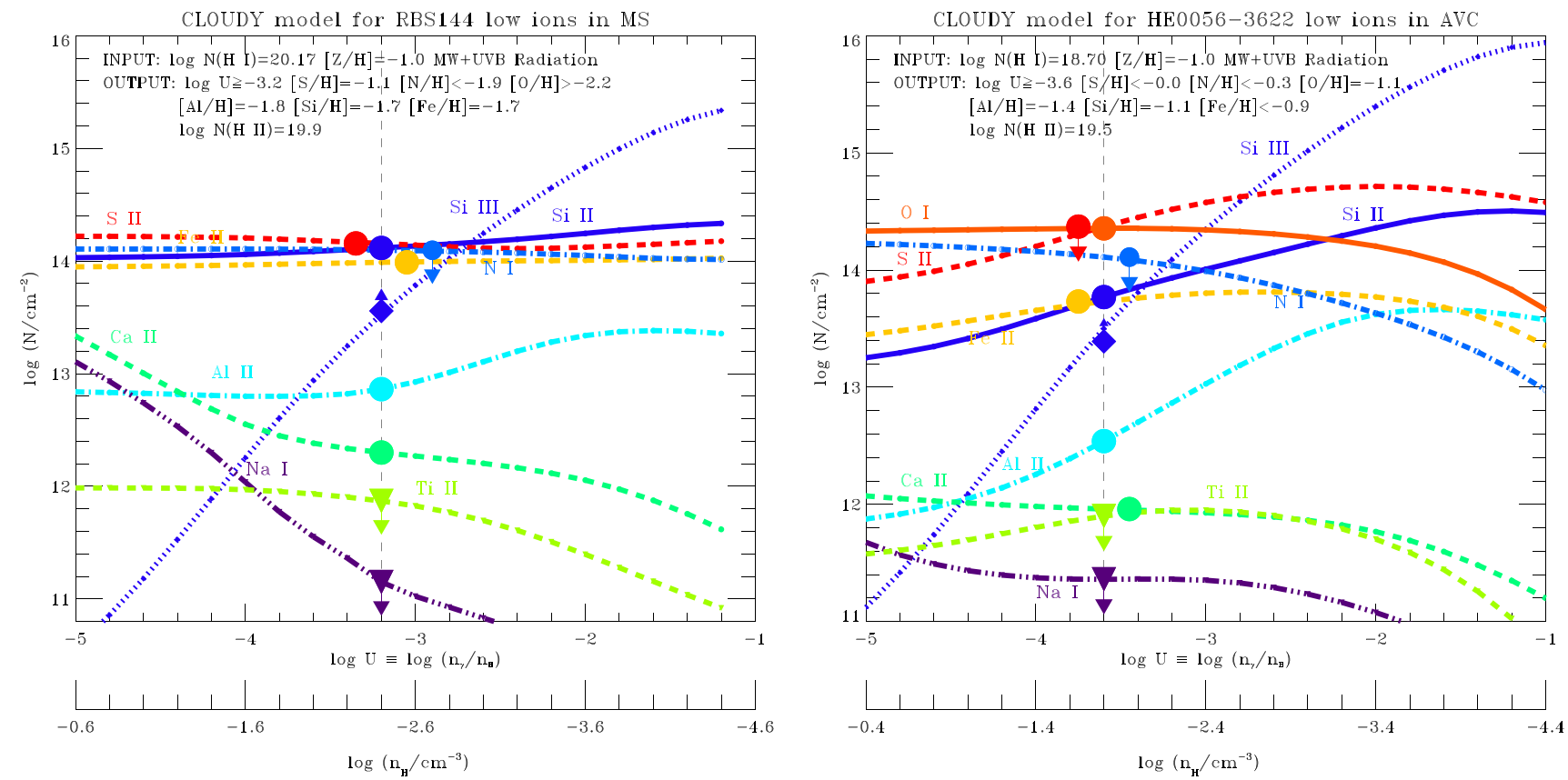

Figure 10. Detailed Cloudy photoionization models of the low-ionization species observed in the MS toward RBS 144 (left), and in the AVCs toward HE 0056-3622 (right). These models assume the low ions exist in a single uniform-density phase exposed to the combined Milky Way plus extragalactic radiation field. The Si III/Si II ratio is used to derive the ionization parameter in the plasma $(\log U \gtrsim-3.2$ for the RBS 144 model and $\log U \gtrsim-3.6$ for the HE 0056-3622 model). The colored lines show the predicted column of each ion, scaled to match the observations at the best-fit $\log U$; the amount by which each line is scaled determines the gas-phase abundance of that element, as annotated on the plot. In turn, the comparison of the gas-phase abundances with the expected solar ratios indicates the depletion of each element onto dust grains. Small offsets have been applied to the observations in the $x$-direction for clarity. See Section 4.3 for details.

(A color version of this figure is available in the online journal.)

In contrast, the derived Ca abundance from the Cloudy model is relatively high, $[\mathrm{Ca} / \mathrm{H}]_{\mathrm{MS}}=-0.3$, even though the Ca II ion abundance is low, [Ca II/HI] $=-2.23 \pm 0.10$, because most of the $\mathrm{Ca}$ is predicted to be in the form of $\mathrm{Ca}^{+2}$ (Ca III). Equivalently, the $\mathrm{Ca}$ II ionization correction in the model is large, $\mathrm{IC}(\mathrm{Ca}$ II $)=+1$.9. If correct, this would correspond to a negative Ca depletion $\delta(\mathrm{Ca})_{\mathrm{MS}}=-0.7$ (i.e., a Ca enhancement relative to S), which would be puzzling for two reasons. First, $\mathrm{Ca}$ and $\mathrm{S}$ are both $\alpha$-elements, so no nucleosynthetic difference in their abundances is expected. Second, in the Galactic ISM, Ca readily depletes onto dust grains (Welty et al. 1996; Savage \& Sembach 1996), so sub-solar $\mathrm{Ca} / \mathrm{S}$ ratios are expected in the gas phase, not super-solar ratios. In another Stream direction (NGC 7469), $\mathrm{F} 10$ found $[\mathrm{Ca} / \mathrm{O}] \approx 0.0$ after applying an ionization correction to the observed $\mathrm{Ca}$ II $/ \mathrm{H}$ I ratio, so the issue is not unique to the RBS 144 direction.

The finding of no apparent $\mathrm{Ca}$ depletion in the Stream in both these directions probably indicates that the single-phase assumption for the low ions, implicit within the Cloudy models, breaks down, and that instead Ca II preferentially traces regions of dense, cold neutral medium which are not properly captured by our Cloudy models, whereas S II traces regions of warm neutral medium and warm ionized medium. The observation that the Ca II absorption in the MS toward RBS 144 occupies a far narrower velocity interval than the other low ions supports this idea. For Na I, the problem is even worse; this ion has an ionization potential of only $5.1 \mathrm{eV}$, so is destroyed easily even in optically thick gas, and is therefore only able to survive in dense clumps. We conclude that caution is necessary when using Cloudy to model Na I and Ca II in HVCs (and other ions whose ionization potential is less than $13.6 \mathrm{eV}$ ), since they likely do not coexist in the same gas phase as the H I and the other low ions (see Paper II and Richter et al. 2005, 2011, for more discussion on this point).

\subsubsection{Cloudy Results toward HE 0056-3622}

Integrating over the full AVC velocity interval $80-200 \mathrm{~km} \mathrm{~s}^{-1}$, we measure a ratio $\log [N(\mathrm{Si}$ III 1206) $/ N(\mathrm{Si}$ II 1526) $]=-0.38$. Matching this ratio gives $\log U \gtrsim-3.6$, corresponding to a bestfit density $\log \left(n_{\mathrm{H}} / \mathrm{cm}^{-3}\right) \lesssim-1.8$ (see Figure $10(\mathrm{~b})$ ). At this $\log U$, the model has an ionized gas column $\log N(\mathrm{H}$ II $)=19.5$, and an ionization fraction $\mathrm{H}$ II $/\left(\mathrm{H}_{\mathrm{I}}+\mathrm{H}\right.$ II $)=86 \%$. If we adopt the ratio $\log [N(\mathrm{Si}$ III $) / N(\mathrm{Si}$ II $)]=-0.90$ measured over the smaller interval 150-200 $\mathrm{km} \mathrm{s}^{-1}$, where both Si III 1206 and Si II 1526 are unsaturated, we derive $\log U=-4.0$ and $\log \left(n_{\mathrm{H}} / \mathrm{cm}^{-3}\right)=$ -1.4 , indicating that the error in the gas density arising because of possible saturation of $\mathrm{Si}$ III 1206 is $\approx 0.4 \mathrm{dex}$.

The model confirms that $[\mathrm{O}$ I/ $\mathrm{HI}]=[\mathrm{O} / \mathrm{H}]$ for all ionization parameters $\log U \lesssim-2.5$, even at the low neutral gas column $\log N(\mathrm{HI})_{\mathrm{AVC}}=18.70$ present in this sightline (see Viegas 1995). The ionization corrections for Al II, Si II, and Fe II at the best-fit $\log U=-3.6$ are more significant in this sightline than toward RBS 144 due to the lower H I column (Figure 9, bottom panel): $\mathrm{IC}(\mathrm{Al}$ II $)=-0.8 \mathrm{dex}, \mathrm{IC}(\mathrm{Si}$ II $)=-0.6 \mathrm{dex}$, and $\mathrm{IC}(\mathrm{Fe}$ II $)=-0.4$ dex. Using these corrections we derive gas-phase abundances $[\mathrm{Al} / \mathrm{H}]_{\mathrm{AVC}}=-1.4,[\mathrm{Si} / \mathrm{H}]_{\mathrm{AVC}}=-1.1$, and $[\mathrm{Fe} / \mathrm{H}]_{\mathrm{AVC}}<-0.9$, corresponding to (small) depletions relative to oxygen of $\delta(\mathrm{Al})_{\mathrm{AVC}} \approx-0.2, \delta(\mathrm{Si})_{\mathrm{AVC}} \approx 0.0$, and $\delta(\mathrm{Fe})_{\mathrm{AVC}}<+0.1$. There is therefore no evidence for dust depletion in the AVC toward HE 0056-3622.

\section{DISCUSSION}

The one-tenth-solar metallicity in the Stream measured from [S II/H I] toward RBS 144 and from [O I/H I] toward NGC 7714 matches the value measured from [O I/H $\mathrm{I}]$ toward NGC 7469 by F10. These measurements are shown in Figure 11, where we plot MS metallicity against angular distance from the center of the SMC for four MS directions observed as part of our HST/ 


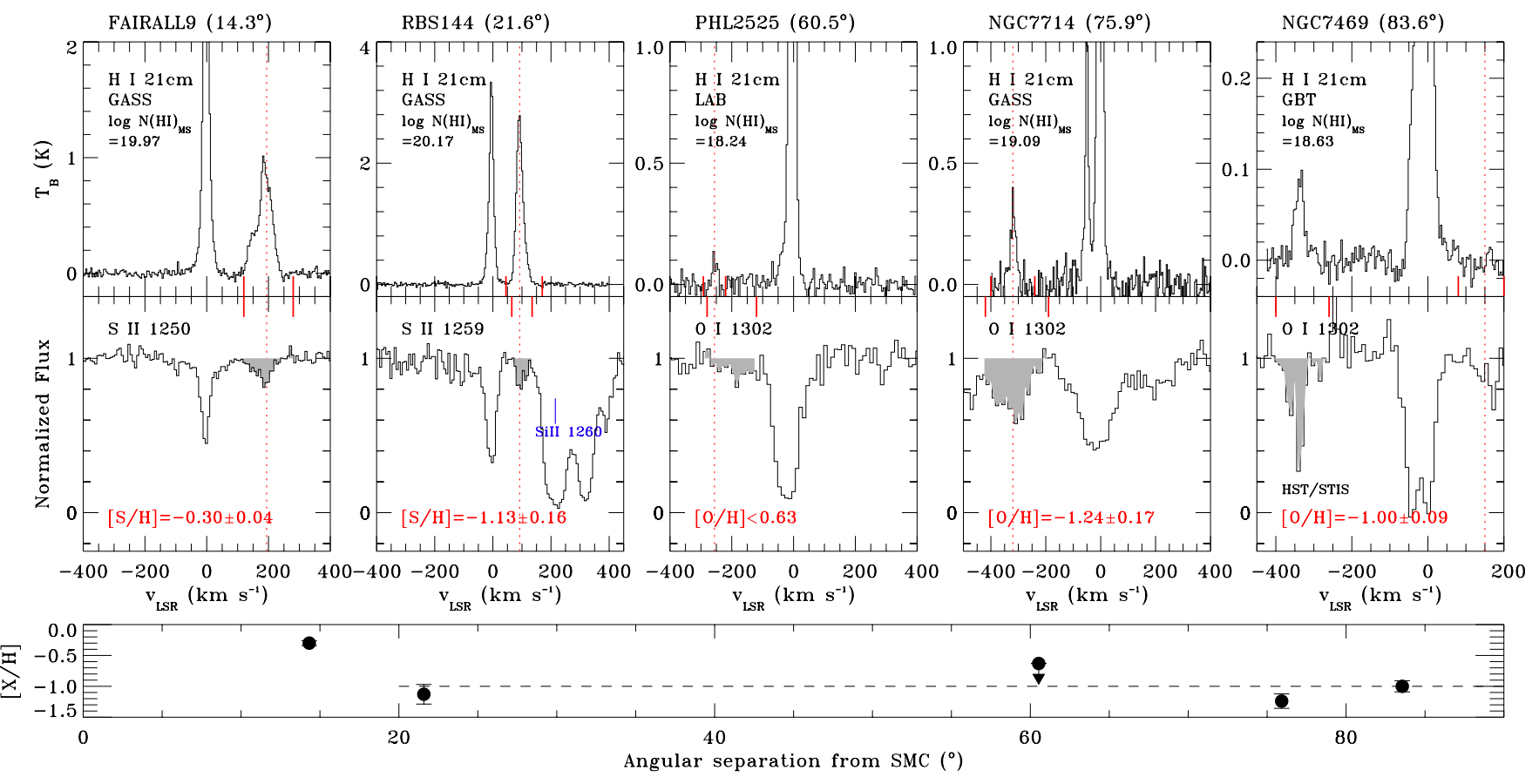

Figure 11. Summary of the metal abundance pattern along the body of the Stream. The upper panels show the GASS, LAB, or Green Bank Telescope (GBT) $21 \mathrm{~cm}$ profiles, giving the H I columns in the MS. The middle panels show the profiles of O I 1302, S II 1259, or S II 1250, giving the metal-line columns. The numbers in parentheses in the title of each column show the angular separation of each direction from the center of the SMC, so the plot is presented in order of increasing separation from the SMC. The lower panel shows the MS abundance $[\mathrm{X} / \mathrm{H}]$ (where $\mathrm{X}$ is $\mathrm{S}$ or $\mathrm{O}$ ) vs. separation from the SMC. The abundance is effectively constant at $\approx 0.1$ solar along the main body of the Stream (shown with the dashed line), until it jumps sharply in the Fairall 9 direction (Paper II). The NGC 7469 data are from F10.

(A color version of this figure is available in the online journal.)

COS program, and one (NGC 7469) observed with HST/STIS. There are therefore three independent measurements of onetenth-solar metallicity along the main body of the MS, and a fourth (PHL 2525) with an upper limit consistent with one-tenth solar. However, these measurements differ from the much higher value $[\mathrm{S} \text { II } / \mathrm{HI}]_{\mathrm{MS}}=-0.30 \pm 0.04(0.5$ solar $)$ measured toward Fairall 9 (Paper II; Gibson et al. 2000), lying only 8.3 away on the sky from RBS 144 , in a direction with a fairly similar MS H I column density (19.95 for Fairall 9 versus 20.17 for RBS 144). This corresponds to an abundance variation of a factor of five over a small scale. However, the Stream's LSR velocity centroid changes by $\approx 100 \mathrm{~km} \mathrm{~s}^{-1}$ between these two directions, from $92 \mathrm{~km} \mathrm{~s}^{-1}$ toward RBS 144 to $194 \mathrm{~km} \mathrm{~s}^{-1}$ toward Fairall 9, indicating that the Stream in the two directions also has very different kinematic properties.

Indeed, if we place our targets on the N08 map of the two bifurcated filaments of the Stream, we find that Fairall 9 lies behind the LMC filament, whereas RBS 144 lies behind the second filament, matching its position both spatially and kinematically (see Figure 1). Thus, our 0.1 solar metallicity measurement in the RBS 144 direction indicates an SMC origin for this second filament (for the reasons given in the next paragraph). The 0.5 solar metallicity measurement in the Fairall 9 direction is consistent with the LMC origin for that filament reported by $\mathrm{N} 08$, although the low $\mathrm{N} / \alpha$ ratio in that sightline and its proximity to both Magellanic Clouds suggests a complex metal enrichment history (Paper II). This is particularly true in light of recent results that both Magellanic Clouds experienced a burst of star formation $\approx 2$ Gyr ago (Weisz et al. 2013).

Our finding that the main body of the Stream has a metal abundance close to 0.1 solar in three directions strongly supports the tidal origin scenario in which most of the Stream was stripped from the SMC $\approx 2$ Gyr ago. This is because (1) the SMC's age-metallicity relation (Pagel \& Tautvaisiene 1998; Harris \& Zaritsky 2004) indicates that its average abundance $2 \mathrm{Gyr}$ ago was $\approx 0.1-0.15$ solar, and (2) the tidal age of the Stream in many numerical studies is $\approx 1.5-2.5 \mathrm{Gyr}$ (Lin et al. 1995; Gardiner et al. 1994; Gardiner \& Noguchi 1996; Connors et al. 2006; Besla et al. 2010, 2012; Diaz \& Bekki 2011a, 2012). That is, the Stream's tidal age and its chemical age are both consistent with an SMC origin. The gas-to-dust ratio measured in the MS toward RBS 144 also agrees with this scenario, lying within a factor of two of the current-day gas-to-dust ratio in the SMC. In contrast, our low MS abundance measurements in all directions except Fairall 9 indicate the main body of the Stream is chemically inconsistent with an LMC origin, since it has been $\approx 5-10$ Gyr since the average LMC abundance was as low as 0.1 solar (Pagel \& Tautvaisiene 1998; Harris \& Zaritsky 2009; Bekki \& Tsujimoto 2012), and the Stream is not that old in any realistic model, except for the regions closest to the Magellanic Clouds which may have been released or polluted more recently. We caution that any metallicity gradients present in the LMC or SMC complicate this interpretation, since lower-metallicity gas at the outskirts of either galaxy will be easier to strip (either by ram pressure or by tidal forces) than gas in the inner regions.

It is worth considering whether some of the difference between the Stream's metallicity in the RBS 144 and Fairall 9 directions could be attributed to small-scale (sub-resolution) structure in $\mathrm{HI}$, which is known to be present in the Stream (Putman et al. 2003b; Stanimirović et al. 2002, 2008; Westmeier \& Koribalski 2008; Nidever et al. 2010). Wakker et al. (2011) have shown that for radio telescopes with beam-sizes of order $10^{\prime}$, variations of up to $20 \%-25 \%$ in $N(\mathrm{H} \mathrm{I})$ determinations can 
occur. In F10, we compared $N(\mathrm{HI})_{\mathrm{MS}}$ determinations toward NGC 7469 from four radio telescopes of beam sizes ranging from $9^{\prime} .1$ to $35^{\prime}$, and found the values varied by $0.15 \operatorname{dex}(40 \%)$. While important, these uncertainties are far less than the factor of five difference in MS metallicity we observe between the RBS 144 and Fairall 9 directions. Furthermore, for RBS 144 the pencil-beam $\mathrm{H}$ i column derived from $\mathrm{Ly} \alpha$ agrees within $2 \sigma$ with the H I column derived from GASS, and so the metallicities derived in this direction do not have a large systematic beamsize error. We conclude that the higher-velocity filament of the MS seen toward Fairall 9 has a genuinely higher metallicity than the lower-velocity filament seen toward RBS 144, despite their proximity on the sky.

Finally, we note that our 0.1 solar metallicity measurements in most of the MS are consistent with the abundances measured in the MB of gas connecting the LMC and SMC. Lehner et al. (2008) measured $[\mathrm{O} / \mathrm{H}]_{\mathrm{MB}}=-0.96_{-0.11}^{+0.13}$ toward O-star DI 1388 , and $[\mathrm{O} / \mathrm{H}]_{\mathrm{MB}}=-1.36$ toward $\mathrm{O}$-star DGIK 975, although these stars appear to lie in front of the principal $\mathrm{H}$ I-emitting phase of the Bridge, so only sample the foreground part of it. Our MS metallicity measurements are also consistent with the results of Misawa et al. (2009), who reported $-1.0<[\mathrm{Z} / \mathrm{H}]_{\mathrm{MB}}<-0.5$ toward QSO PKS 0312-770, the sightline to which samples the full radial extent of the Bridge. However, we note that in recent simulations (Besla et al. 2012; Diaz \& Bekki 2012) the Bridge and the Stream are created at different points in time, so their similar present-day metallicities may be partly coincidental.

\section{SUMMARY}

In this first paper of a series presenting combined $H S T / \mathrm{COS}$ and VLT/UVES spectroscopy of the MS, we have investigated the Stream's chemical abundances using spectra of four AGN: RBS 144, PHL 2525, NGC 7714, and HE 0056-3622. These sightlines all lie behind the main body of the Stream, and sample a wide range of $\mathrm{H}$ I column density, from $\log N(\mathrm{HI})_{\mathrm{MS}}=20.17$ toward RBS 144 down to 18.24 toward PHL 2525. Three of these targets (RBS 144, PHL 2525, and NGC 7714) have UV spectra that allow MS abundance measurements. The fourth (HE 0056-3622) cannot be used for this purpose since the MS velocity centroid in this direction $\left(-10 \mathrm{~km} \mathrm{~s}^{-1}\right)$ overlaps with foreground Galactic absorption; however, we have measured the AVC absorption centered at $150 \mathrm{~km} \mathrm{~s}^{-1}$ in that sightline. A wide range of metal-line species is detected in the Stream including O I, C II, C Iv, Si II, Si III, Si IV, S II, Al II, Fe II, and Ca II. Our main results are as follows.

1. Toward RBS 144, we measure a MS sulfur abundance $[\mathrm{S} / \mathrm{H}]_{\mathrm{MS}}=[\mathrm{S} \mathrm{II} / \mathrm{HI}]_{\mathrm{MS}}=-1.13 \pm 0.16$. Toward NGC 7714, we measure a similar value $[\mathrm{O} / \mathrm{H}]_{\mathrm{MS}}=[\mathrm{O} \mathrm{I} /$ $\mathrm{HI}]_{\mathrm{MS}}=-1.24 \pm 0.20$. Toward PHL 2525, we measure an upper limit $[\mathrm{O} / \mathrm{H}]_{\mathrm{MS}}<-0.63(3 \sigma)$, based on a nondetection in O I 1302. When combining these new measurements with the published metallicity toward NGC 7469 (F10), there are now three good measurements along the main body of the MS consistent with $\approx 0.1$ solar metallicity. This matches the SMC's abundance $\approx 2$ Gyr ago, given its age-metallicity relation (Pagel \& Tautvaisiene 1998; Harris \& Zaritsky 2004). Tidal origin models for the Stream independently age it at $\approx 1.5-2.5$ Gyr (e.g., Gardiner et al. 1994; Connors et al. 2006; Diaz \& Bekki 2011a, 2012; Besla et al. 2012). Furthermore, the gas-to-dust ratio we measure in the Stream toward RBS 144 is within a factor of two of the average value found in the diffuse gas of the
SMC. Thus we conclude based on chemical evidence that most of the Stream originated in the SMC about 2 Gyr ago. We note that the RBS 144 sightline passes through the second (non-LMC) filament identified in $\mathrm{H}_{\mathrm{I}} 21 \mathrm{~cm}$ emission by N08, and thus our metallicity measurements resolve the nature of this filament's origin (in the SMC).

2. The Stream's $[\mathrm{S} / \mathrm{H}]$ abundance we measure toward RBS 144 differs by $\approx 0.7$ dex (a factor of five) from the value $[\mathrm{S} / \mathrm{H}]_{\mathrm{MS}}=-0.30 \pm 0.04$ measured toward Fairall 9 (only 8.3 away) in Paper II of this series. Fairall 9 lies behind the LMC filament of the Stream (N08). Our Cloudy simulations indicate that ionization corrections are not the reason for the different abundances: we find that the ionization correction $\mathrm{IC}(\mathrm{S}$ II $)=[\mathrm{S} / \mathrm{H}]-[\mathrm{S} \mathrm{II} / \mathrm{H} \mathrm{I}] \approx 0.07 \mathrm{dex}$ at the best-fit density toward RBS 144 , and $\left|\mathrm{IC}\left(\mathrm{S}_{\mathrm{II}}\right)\right|<0.01 \mathrm{dex}$ toward Fairall 9, indicating the difference in the abundances is real. Furthermore, the Stream exhibits very different gasto-dust ratios and velocity centroids in the two directions. This shows that the bifurcation of the Stream is seen not only in its spatial extent and its kinematics, but also in its chemical enrichment.

3. We find evidence for dust depletion in the Stream in the form of sub-solar $\mathrm{Si} / \mathrm{S}, \mathrm{Al} / \mathrm{S}$, and $\mathrm{Fe} / \mathrm{S}$ ratios toward RBS 144, where $\mathrm{S}$ is used as an undepleted reference element. Toward RBS 144, we measure depletion levels of $\delta(\mathrm{Al})_{\mathrm{MS}} \approx-0.7$, $\delta(\mathrm{Si})_{\mathrm{MS}} \approx-0.6$, and $\delta(\mathrm{Fe})_{\mathrm{MS}} \approx-0.6$. These depletions are similar to those found in the Leading Arm by Lu et al. (1998), and indicate that dust grains survive the process that ejected the Stream from the Magellanic Clouds.

4. Toward HE 0056-3622, we measure an oxygen abundance $[\mathrm{O} / \mathrm{H}]_{\mathrm{AVC}}=[\mathrm{OI} / \mathrm{HI}]_{\mathrm{AVC}}=-1.03 \pm 0.18$ in the cloud centered at $v_{\mathrm{LSR}}=150 \mathrm{~km} \mathrm{~s}^{-1}$, which belongs to a population of AVCs found near the south Galactic pole. The similarity between this metallicity and the MS metallicity suggests the AVCs are associated with the Stream rather than tracing foreground Galactic material. However, we cannot rule out the possibility that they are associated with more distant material, e.g., in the Sculptor Group, which lies in this direction at similar velocities (Mathewson et al. 1975; Putman et al. 2003b).

A detailed study of the Stream's chemical abundances in the Fairall 9 direction is presented in Paper II. In Paper III we will address the Stream's ionization level and fate, and the more general question of the role of tidal streams in fueling $L_{*}$ galaxies.

We gratefully thank Justin Ely for providing a night-only reduction of the COS data, and we acknowledge valuable conversations with Julia Roman-Duval, David Nidever, Gurtina Besla, Karl Gordon, Mary Putman, and Kat Barger. We are grateful to the referee for a useful and insightful report. Support for program No. 12604 was provided by NASA through a grant from the Space Telescope Science Institute, which is operated by the Association of Universities for Research in Astronomy, Inc., under NASA contract NAS 5-26555.

\section{REFERENCES}

Asplund, M., Greenness, N., Jacques Sauval, A., \& Scott, P. 2009, ARA\&A, 47, 481

Ballester, P., Modigliani, A., Boitquin, O., et al. 2000, Msngr, 101, 31 Bekki, K., \& Tsujimoto, T. 2012, ApJ, 761, 180

Besla, G., Kallivayalil, N., Hernquist, L., et al. 2010, ApJL, 721, L97 Besla, G., Kallivayalil, N., Hernquist, L., et al. 2012, MNRAS, 421, 2109 
Bland-Hawthorn, J., \& Maloney, P. R. 1999, ApJL, 510, L33

Bland-Hawthorn, J., \& Maloney, P. R. 2002, in ASP Conf. Ser. 254, Extragalactic Gas at Low Redshift, ed. J. S. Mulchaey \& J. Stocke (San Francisco, CA: ASP), 267

Bland-Hawthorn, J., Sutherland, R., Agertz, O., \& Moore, B. 2007, ApJL, 670, L109

Brüns, C., Kerp, J., Staveley Smith, L., et al. 2005, A\&A, 432, 45

Connors, T. W., Kawata, D., \& Gibson, B. K. 2006, MNRAS, 371, 108

Dekker, H., D’Odorico, S., Kaufer, A., Delabre, B., \& Kotzlowski, H. 2000, Proc. SPIE, 4008, 534

Diaz, J. D., \& Bekki, K. 2011a, MNRAS, 413, 2015

Diaz, J. D., \& Bekki, K. 2011b, PASA, 28, 117

Diaz, J. D., \& Bekki, K. 2012, ApJ, 750, 36

Dickey, J. M., \& Lockman, F. J. 1990, ARA\&A, 28, 215

Dieter, N. H. 1971, A\&A, 12, 59

Draine, B. T. 2003, ARA\&A, 41, 241

Ferland, G. J., Korista, K. T., Verner, D. A., et al. 1998, PASP, 110, 761

Fitzpatrick, E. L. 1996, ApJL, 473, L55

Fox, A. J., Savage, B. D., \& Wakker, B. P. 2005a, AJ, 130, 2418

Fox, A. J., Wakker, B. P., Savage, B. D., et al. 2005b, ApJ, 630, 332

Fox, A. J., Wakker, B. P., Smoker, J. V., et al. 2010, ApJ, 718, 1046 (F10)

Fujimoto, M., \& Sofue, Y. 1976, A\&A, 47, 263

Gardiner, L. T., \& Noguchi, M. 1996, MNRAS, 278, 191

Gardiner, L. T., Sawa, T., \& Fujimoto, M. 1994, MNRAS, 266, 567

Gibson, B. K., Giroux, M. L., Penton, S. V., et al. 2000, AJ, 120, 1803

Gordon, K. D., Bot, C., Muller, E., et al. 2009, ApJL, 690, L76

Green, J. C., Froning, C. S., Osterman, S., et al. 2012, ApJ, 744, 60

Haardt, F., \& Madau, P. 2012, ApJ, 746, 125

Harris, J., \& Zaritsky, D. 2004, AJ, 127, 1532

Harris, J., \& Zaritsky, D. 2009, AJ, 138, 1243

Haynes, M. P., \& Roberts, M. S. 1979, ApJ, 227, 767

Heitsch, F., \& Putman, M. E. 2009, ApJ, 698, 1485

Howk, J. C., \& Consiglio, S. M. 2012, ApJ, 759, 97

Howk, J. C., Sembach, K. R., \& Savage, B. D. 2006, ApJ, 637, 333

Hulsbosch, A. N. M., \& Wakker, B. P. 1988, A\&AS, 75, 191

Jenkins, E. B. 2009, ApJ, 700, 1299

Jensen, A. G., Rachford, B. L., \& Snow, T. P. 2005, ApJ, 619, 891

Kalberla, P. M. W., Burton, W. B., Hartmann, D., et al. 2005, A\&A, 440, 775

Kalberla, P. M. W., McClure-Griffiths, N. M., Pisano, D. J., et al. 2010, A\&A, 521,17

Kallivayalil, N., van der Marel, R. P., \& Alcock, C. 2006a, ApJ, 652, 1213

Kallivayalil, N., van der Marel, R. P., Alcock, C., et al. 2006b, ApJ, 638, 772

Kallivayalil, N., van der Marel, R. P., Besla, G., Anderson, J., \& Alcock, C. 2013, ApJ, 764, 161

Lehner, N. 2002, ApJ, 578, 126

Lehner, N., Howk, J. C., Keenan, F. P., \& Smoker, J. V. 2008, ApJ, 678, 219

Lehner, N., Sembach, K. R., Dufton, P. L., Rolleston, W. R. J., \& Keenan, F. P. 2001, ApJ, 551, 781

Leroy, A., Bolatto, A., Stanimirović, S., et al. 2007, ApJ, 658, 1027

Lin, D. N. C., Jones, B. F., \& Klemola, A. R. 1995, ApJ, 439, 652

Lin, D. N. C., \& Lynden-Bell, D. 1982, MNRAS, 198, 707

Lu, L., Savage, B. D., \& Sembach, K. R. 1994, ApJL, 437, L119
Lu, L., Savage, B. D., Sembach, K. R., et al. 1998, AJ, 115, 162

Mastropietro, C., Moore, B., Mayer, L., Wadsley, J., \& Stadel, J. 2005, MNRAS, 363, 509

Mathewson, D. S., Cleary, M. N., \& Murray, J. D. 1974, ApJ, 190, 291

Mathewson, D. S., Cleary, M. N., \& Murray, J. D. 1975, ApJL, 195, L97

Matthews, D., Staveley-Smith, L., Dyson, P., \& Muller, E. 2009, ApJL, 691, L115

McClure-Griffiths, N. M., Pisano, D. J., Calabretta, M. R., et al. 2009, ApJS, 181,398

Meurer, G. R., Bicknell, G. V., \& Gingold, R. A. 1985, PASAu, 6, 195

Meyer, D. M., Jura, M., \& Cardelli, J. A. 1998, ApJ, 493, 222

Misawa, T., Charlton, J. C., Kobulnicky, H. A., Wakker, B. P., \& BlandHawthorn, J. 2009, ApJ, 695, 1382

Moore, B., \& Davis, M. 1994, MNRAS, 270, 209

Morton, D. C. 2003, ApJS, 149, 205

Murai, T., \& Fujimoto, M. 1980, PASJ, 32, 581

Nidever, D. L., Majewski, S. R., \& Burton, W. B. 2008, ApJ, 679, 432 (N08)

Nidever, D. L., Majewski, S. R., Burton, W. B., \& Nigra, L. 2010, ApJ, 723, 1618

Nigra, L., Stanimirović, S., Gallagher, J. S., et al. 2012, ApJ, 760, 48

Olano, C. A. 2004, A\&A, 423, 895

Pagel, B. E. J., \& Tautvaisiene, G. 1998, MNRAS, 299, 535

Piatek, S., Pryor, C., \& Olszewski, E. W. 2008, AJ, 135, 1024

Putman, M. E., Bland-Hawthorn, J., Veilleux, S., et al. 2003a, ApJ, 597, 948

Putman, M. E., Peek, J. E. G., \& Joung, M. R. 2012, ARA\&A, 50, 491

Putman, M. E., Staveley-Smith, L., Freeman, K. C., Gibson, B. K., \& Barnes, D. G. 2003b, ApJ, 586, 170

Richter, P., Fox, A. J., Wakker, B. P., et al. 2013, ApJ, 772, 111 (Paper II)

Richter, P., Krause, F., Fechner, C., Charlton, J. C., \& Murphy, M. T. 2011, A\&A, 528, A 12

Richter, P., Sembach, K. R., Wakker, B. P., \& Savage, B. D. 2001, ApJL, 562, L181

Richter, P., Westmeier, T., \& Brüns, C. 2005, A\&A, 442, L49

Russell, S. C., \& Dopita, M. A. 1992, ApJ, 384, 508

Savage, B. D., \& Sembach, K. R. 1991, ApJ, 379, 245

Savage, B. D., \& Sembach, K. R. 1996, ARA\&A, 34, 279

Sembach, K. R., Howk, J. C., Savage, B. D., \& Shull, J. M. 2001, AJ, 121, 992

Sembach, K. R., Wakker, B. P., Savage, B. D., et al. 2003, ApJS, 146, 165

Sofia, U. J., Gordon, K. D., Clayton, G. C., et al. 2006, ApJ, 636, 753

Stanimirović, S., Dickey, J. M., Krčo, M., \& Brooks, A. M. 2002, ApJ, 576, 773

Stanimirović, S., Hoffman, S., Heiles, C., et al. 2008, ApJ, 680, 276

Viegas, S. 1995, MNRAS, 276, 268

Wakker, B. P., Lockman, F. J., \& Brown, J. M. 2011, ApJ, 728, 159

Wakker, B. P., Oosterloo, T. A., \& Putman, M. E. 2002, AJ, 123, 1953

Wannier, P., \& Wrixon, G. T. 1972, ApJL, 173, L119

Weiner, B. J., \& Williams, T. B. 1996, AJ, 111, 1156

Weisz, D. R., Dolphin, A. E., Skillman, E. D., et al. 2013, MNRAS, 431, 364

Welty, D. E., Lauroesch, J. T., Blades, J. C., Hobbs, L. M., \& York, D. G. 1997, ApJ, 489, 672

Welty, D. E., Morton, D. C., \& Hobbs, L. M. 1996, ApJS, 106, 533

Westmeier, T., \& Koribalski, B. S. 2008, MNRAS, 388, L29

Wolfe, A. M., Prochaska, J. X., \& Gawiser, E. 2003, ApJ, 593, 215 